\title{
Dislocation Effects in Smectic-A Liquid Crystals
}

\section{Citation}

Pershan, Peter S. 1974. Dislocation effects in smectic-A liquid crystals. Journal of Applied Physics 45(4): 1590-1604.

\section{Published Version}

doi:10.1063/1.1663462

\section{Permanent link}

http://nrs.harvard.edu/urn-3:HUL.InstRepos:10361965

\section{Terms of Use}

This article was downloaded from Harvard University's DASH repository, and is made available under the terms and conditions applicable to Other Posted Material, as set forth at http:// nrs.harvard.edu/urn-3:HUL.InstRepos:dash.current.terms-of-use\#LAA

\section{Share Your Story}

The Harvard community has made this article openly available.

Please share how this access benefits you. Submit a story.

Accessibility 


\title{
Dislocation effects in smectic- $A$ liquid crystals*
}

\author{
P. S. Pershan \\ Division of Engineering and Applied Physics, Harvard University, Cambridge, Massachusetts 02138 \\ (Received 5 November 1973) \\ A method for calculating stress-strain fields around edge dislocations in smectic- $A$ samples is \\ discussed. In large part the method is isomorphic with the formalism for calculating magnetic fields \\ around lines of electric current. The force law between dislocations that follows from the analogy is \\ equivalent to the accepted force law between dislocations in crystals. In addition to rederiving the \\ expression for the strain field surrounding an isolated edge dislocation that was first given by de \\ Gennes, we present the solutions for the stress-strain fields surrounding dislocations near one or two \\ boundaries and also the strain field surrounding an edge dislocation that is curved to form a circular \\ loop. The stress-strain fields surrounding other defects with the same symmetries and boundary \\ conditions can be expressed in terms of the above-mentioned solutions using Green's function \\ techniques. The relative stability of dislocations in samples with different types of boundaries and also \\ the effects of dislocations on the elastic properties of smectic samples are also discussed in some \\ detail. We comment briefly on the relation between the analogy discussed here and an earlier one \\ developed by de Gennes.
}

\section{INTRODUCTION}

Recent interest in the physical properties of liquid crystals, particularly of the smectic type, warrants the same type of detailed understanding of the effects of dis locations on these systems as is currently available for conventional crystals. ${ }^{1,2}$ Although there have been studies directed toward this end, see for example the work of Friedel and Kleman ${ }^{3}$ and Bouligand, ${ }^{4}$ there remain further questions concerning the effects of dislocations in liquid crystals that we cannot yet answer. For example, in the presence of dislocations the existing hydrodynamic theories of liquid crystals are not strictly valid. ${ }^{5,6}$ We require criteria for when dislocation effects can or cannot be neglected and the conditions under which the hydrodynamics are applicable. The analogy developed by de Gennes ${ }^{7}$ between superfluids and smectics, together with the observation that dislocations in smectics are analogous to vortices in superfluids, ${ }^{8}$ suggest the type of effects that might be expected.

Although focal conic-type defects do occur in smectic samples under a variety of conditions; it does seem that single-domain planar samples can be prepared without the focal conic structure. For these samples simple topological considerations suggest the possible occur-

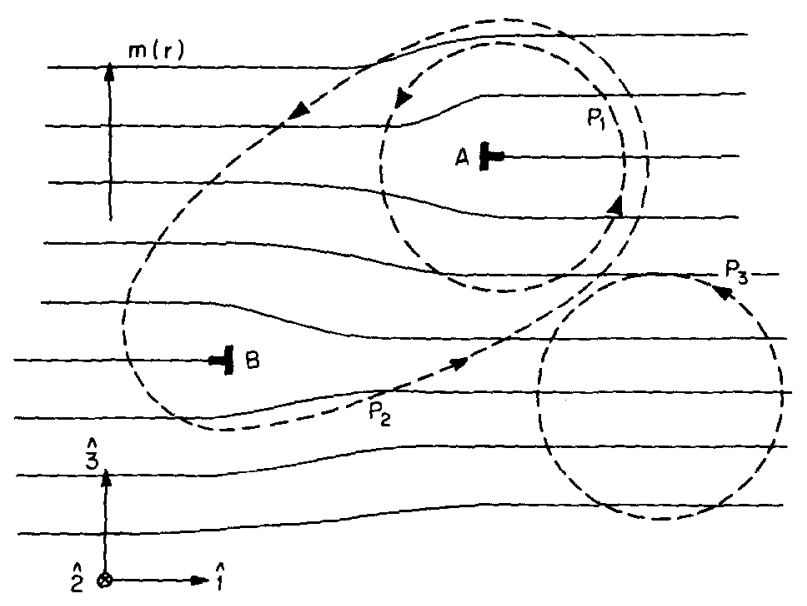

FIG. 1. Examples to illustrate the sign convention of the path integrals, Eqs. (2.2) and (2.3). rence of dislocations and there is some experimental evidence supporting this. ${ }^{3,4}$ Furthermore, Bouligand ${ }^{4}$ has argued that focal conic defects are not sufficient to explain observed textures in smectics and that dislocations must be present. More recently Clark and Meyer ${ }^{9}$ proposed dislocation effects to account for the low-frequency relaxation in recently observed light scattering phenomena. ${ }^{10,11}$ Durand ${ }^{12}$ and also de Gennes ${ }^{13}$ have discussed how defects in the smectic layers would influence light-scattering cross sections. de Gennes even presented one specific solution for the strain pattern surrounding an edge dislocation in the smectic $-A$ structure.

In this paper we will further analyze the stress-strain patterns surrounding edge dislocations in smectic- $A$ liquid crystals subjected to specific boundary conditions and externally applied forces. Forces of one dislocation on another, and also the forces of boundaries on dislocations are calculated from the stress field using the Peach-Koehler result. ${ }^{14,15}$ In Sec. II we review that result as it is applicable to smectic- $A$ liquid crystals and discuss an analogy between the smectic-dislocation problem and the interactions between magnetic fields and electric currents. In Sec. III we rederive de Gennes's result ${ }^{13}$ for the strain field surrounding an edge dislocation and calculate the resultant stress field. In Sec. IV we consider a pair of dislocations, the mutual forces between them, and the energy of interaction between the pair. In Sec. $V$ we discuss the interaction of edge dislocations with some simple boundaries and in Sec. VI we discuss thin smectic samples and the influence of two boundaries on the stability of dislocations. In Sec. VII we discuss wedge-shaped samples and the relative stability of arrays of dislocations. In Sec. VIII we present exact solutions for the stress-strain pattern surrounding a circular dislocation loop and discuss the effects of such loops on bulk elastic measurements. In Sec. IX we comment on screw dislocations. Finally in Sec. $\mathrm{X}$ we discuss possibilities for further theoretical work relative to the macroscopic elastic properties of smectic samples.

\section{FORMAL DEVELOPMENT}

The smectic- $A$ phase is such that one can define a vector field $\mathbf{m}(r)$ with the property that

Copyright $\odot 1974$ American Institute of Physics 


$$
\int_{P\left(\mathbf{r}_{1}, \mathbf{r}_{2}\right)} d \mathrm{r} \cdot \mathrm{m}(r)=\delta N_{P}\left(\mathbf{r}_{1}, \mathbf{r}_{2}\right),
$$

where $P\left(\mathbf{r}_{1}, \mathbf{r}_{2}\right)$ specifies the path for the line integral between points $r_{1}$ and $r_{2}$ and $\delta N_{P}\left(r_{1}, r_{2}\right)$ is equal to the number of smectic layers traversed by $P\left(r_{1}, r_{2}\right){ }^{16}$ The quantity $\delta N_{P}\left(\mathbf{r}_{1}, \mathbf{r}_{2}\right)$ plays a role similar to the phase variable de Gennes introduced in his analogy to the superfluid. ${ }^{7}$ There, and here also, it is necessary to assume that one can define a direction of increasing phase. Thus, if $d \mathbf{r}$ has a component in the direction of increasing phase, $d \mathbf{r} \cdot \mathrm{m}(r)>0$, otherwise it is negative. If $P\left(\mathbf{r}_{1}, \mathbf{r}_{2}\right)$ is some closed path,

$$
\oint_{P} d \mathbf{r} \cdot \mathbf{m}(r)=N_{P}
$$

where $N_{P}=0, \pm 1, \pm 2, \ldots$ depending on the number and type of defects enclosed in $P$. Figure 1 illustrates some examples. The direction of "phase" increase is fixed by $\mathbf{m}(r)$. Note that $\mathbf{m}(r)$ is locally normal to the smectic layers ${ }^{17}$ and we take the $\hat{3}$ direction to define the average direction for $\mathrm{m}(r)$. For path $P_{1}, N_{P_{1}}=+1$ while for paths $P_{2}$ and $P_{3}, N_{P_{2}}=N_{P_{3}}=0$. A path similar to $P_{1}$ but surrounding only $B$ would result in an $N_{P}=-1$. The quantity $N_{p}$ is equal to the net number of dislocations (i.e., positive minus negative) enclosed in the path. Consider only dislocations of unit strength, we assign to each a unit vector $\hat{l}$ along the dislocation line subject to the condition that a path $P$ enclosing only that dislocation in a "right-hand sense" relative to $\hat{l}$ obtains $N_{P}=+1$. Thus for dislocation $A, \hat{l}_{A}$ is out of the page and for $B$, $\hat{l}_{B}$ is into the page. Formally we can define a density of dislocation lines per unit area $I(r)$ such that

$$
\oint d \mathbf{r} \cdot \mathbf{m}(\mathbf{r})=\int d \mathbf{A} \cdot \mathbf{l}(r)
$$

or

$$
\nabla \times \mathrm{m}(r)=\mathrm{l}(\mathbf{r}) \text {. }
$$

In the absence of either dislocations or externally applied strains $\mathrm{m}(\mathbf{r})=\hat{z} / a$, where $a$ is the average equilibrium thickness of one smectic layer. Deviations of $m(r)$ from this value define the local strain. Away from the dislocation, one may assume that the deviations are small and express $\delta \mathrm{m}(\mathrm{r})$ in terms of the hydrodynamic variable $\mu$ describing the layer displacements ${ }^{18_{0}}$.

$$
\delta m(r)_{i}=-a^{-1} \partial_{i} \delta \mu(r) .
$$

At constant pressure and temperature the local energy density associated with $\delta \mu(r)$ is given by ${ }^{19}$

$$
\delta \epsilon=\phi_{i} \partial_{i} \delta \mu
$$

or in terms of $\delta \mathrm{m}(r)$,

$$
\delta \epsilon=-a \phi_{i} \delta m_{i} \text {. }
$$

de Gennes's ${ }^{20}$ expression for the energy density

$$
\epsilon=\frac{1}{2} b\left(\partial_{3} \mu\right)^{2}+\frac{1}{2} K\left[\left(\partial_{11}+\partial_{22}\right) \mu\right]^{2}
$$

can be written ${ }^{21}$

$$
\epsilon=\frac{1}{2} a^{2} b\left(\delta m_{3}\right)^{2}+\frac{1}{2} K a^{2}\left(\partial_{1} \delta m_{1}+\partial_{2} \delta m_{2}\right)^{2}
$$

and

$$
\begin{aligned}
& \phi_{3}=-a b \delta m_{3}, \\
& \phi_{1}=+K a\left(\partial_{11} \delta m_{1}+\partial_{12} \delta m_{2}\right), \\
& \phi_{2}=+K a\left(\partial_{22} \delta m_{2}+\partial_{12} \delta m_{1}\right) .
\end{aligned}
$$

If the smectic is in mechanical equilibrium and the velocity of the smectic is zero, Eq. (2.22) of Ref. 5 gives at constant pressure

$$
\partial_{i} \phi_{i}=0 \text {. }
$$

Equations (2.3b), (2.5b), and (2.8) are isomorphic with the equations describing the magnetic fields surrounding lines of electric current. The vector field $\mathrm{m}$ is analogous to the magnetic field $\mathrm{H}, a \phi$ is analogous to $(4 \pi)^{-1} B$, and 1 is analogous to $(4 \pi / c) J(r)$, where $J(r)$ is the free-carrier current density. This analogy is most profitable for calculating interaction effects between relatively isolated dislocations. Equation (2.3b) could also be considered analogous to the London equation $\left[\nabla \times \mathrm{J}_{s}=-\left(n_{s} e^{2} / m c\right) \mathrm{B}\right]$ if $\delta \mathrm{m}$ is associated with the supercurrent $J_{s}$ and the dislocation density $I$ is associated with B. The latter analogy was introduced by de Gennes. ${ }^{7}$ The two analogies do not conflict with each other, but rather both are useful for understanding different aspects of smectics.

It is well known that a system of wires carrying fixed electric currents cannot be in stable mechanical equilibrium if the only forces are the classical electromagnetic forces due to fixed currents. Nevertheless, the electromagnetic forces between the wires can be calculated from the electromagnetic part of the free energy ${ }^{22}$ using the principle of virtual work. In order for such a collection of current-carrying wires to be in mechanical equilibrium, there must be nonelectromagnetic forces to cancel the electromagnetic force density

$$
F=(1 / c) J \times B \text {. }
$$

On the basis of the above-mentioned analogy, one can define the "force density" on an array of dislocations as

$$
\mathbf{F}=a \mathbf{l} \times \phi \text {. }
$$

Although the question of what one means precisely by the force on a dislocation does not have an obvious answer, it has been carefully considered in the case of crystals. ${ }^{23}$ Equation (2.10) is just the Peach-Koehler ${ }^{24,25}$ equation under conditions of zero pressure. ${ }^{26}$ In order for a par ticular array of dislocations to be in mechanical equilibrium, there will necessarily have to be other forces (as in the magnetic problem) to stabilize the system. In the dislocation problem there can be, for example, the line tension of a dislocation core, local pinning effects due to surface irregularities or impurities. In any event, as in the magnetic problem we can consider the force [Eq. (2.10)] analogously to the force (2.9) in the magnetic problem.

\section{STRESS-STRAIN PATTERN SURROUNDING AN ISOLATED DISLOCATION}

Consider a dislocation of the type $A$ as in Fig. 1 at the position $\left(x_{1}, x_{3}\right)=(0,0)$ and of indefinite extent in the $x_{2}$ direction. Assume also that the sample is of infinite extent in the $x_{1}$ and $x_{3}$ directions and that boundary effects can be neglected (we will discuss boundary effects later). Equation (2.3b) becomes

$$
\partial_{3} \delta m_{1}-\partial_{1} \delta m_{3}=-\delta\left(x_{1}\right) \delta\left(x_{3}\right)
$$

and Eq. (2.8) [using (2.7)] becomes

$$
-a b \partial_{3} \delta m_{3}+K a\left(\partial_{1}\right)^{3} \delta m_{1}=0 \text {. }
$$


Elimination of $\delta m_{3}$ from Eq. (3.1) gives

$$
\partial_{33} \delta m_{1}-\lambda^{2}\left(\partial_{1}\right)^{4} \delta m_{1}=-\delta\left(x_{1}\right) \partial_{3} \delta\left(x_{3}\right)
$$

where $\lambda \equiv(K / b)^{1 / 2}$. Taking the Fourier transform and inverting gives

$\delta m_{1}\left(x_{1}, x_{3}\right)=\frac{1}{4 \pi^{2}} \iint \frac{i q_{3}}{q_{3}^{2}+\lambda^{2} q_{1}^{4}} \exp \left[i\left(q_{1} x_{1}+q_{3} x_{3}\right)\right] d q_{1} d q_{3}$,

or

$\delta m_{1}\left(x_{1}, x_{3}\right)=-\left[4(\pi \lambda)^{1 / 2}\right]^{-1} x_{3}\left|x_{3}\right|^{-3 / 2} \exp \left[-x_{1}^{2}\left(4 \lambda\left|x_{3}\right|\right)^{-1}\right]$.

de Gennes first obtained this result ${ }^{13}$ in terms of the angle $\delta \theta$ between the local normal to the smectic layers and the $\hat{z}$ direction, $\delta \theta=-\partial_{1} \mu=a \delta m_{1}$. The layer dilation $\delta a / a=-a \delta m_{3}$,

$$
\delta m_{3}\left(x_{1}, x_{3}\right)=+\lambda x_{3}\left|x_{3}\right|^{-1} \partial_{1} \delta m_{1}\left(x_{1}, x_{3}\right) \text {. }
$$

The components of stress field $\phi_{1}$ and $\phi_{3}$ due directly to the dislocation are obtained by substituting Eqs. $(3,3)$ into Eqs. $(2,7)$.

Although the stress tensor itself is not uniquely defined, any choice yielding the same acceleration $\left(\dot{g}_{i}\right.$ $\left.=-\partial_{j} \sigma_{i j}\right)$ is acceptable; one choice gives

$$
\begin{aligned}
& \sigma_{33}=a b \delta m_{3}=-\phi_{3}, \\
& \sigma_{13}=-a K \partial_{13} m_{1}=-\phi_{1}, \\
& \sigma_{11}=a K \partial_{13} m_{1} .
\end{aligned}
$$

Substitution of $\mathrm{Eq}$. (3.3b) gives

$$
\begin{aligned}
\sigma_{33}= & a b\left[8(\pi \lambda)^{1 / 2}\right]^{-1} x_{1}\left|x_{3}\right|^{-3 / 2} \exp \left[-x_{1}^{2}\left(4 \lambda\left|x_{3}\right|\right)^{-1}\right], \\
\sigma_{13}= & -\lambda a b\left[8(\pi \lambda)^{1 / 2}\right]^{-1} x_{3}\left|x_{3}\right|^{-5 / 2}\left[1-x_{1}^{2}\left(2 \lambda\left|x_{3}\right|\right)^{-1}\right] \\
& \times \exp \left[-x_{1}^{2}\left(4 \lambda\left|x_{3}\right|\right)^{-1}\right], \\
\sigma_{11}= & \lambda a b(\pi \lambda)^{-1 / 2} x_{1}\left|x_{3}\right|^{-5 / 2}\left[-\frac{3}{2}+x_{1}^{2}\left(4 \lambda\left|x_{3}\right|\right)^{-1}\right] \\
& \times \exp \left[-x_{1}^{2}\left(4 \lambda\left|x_{3}\right|\right)^{-1}\right] .
\end{aligned}
$$

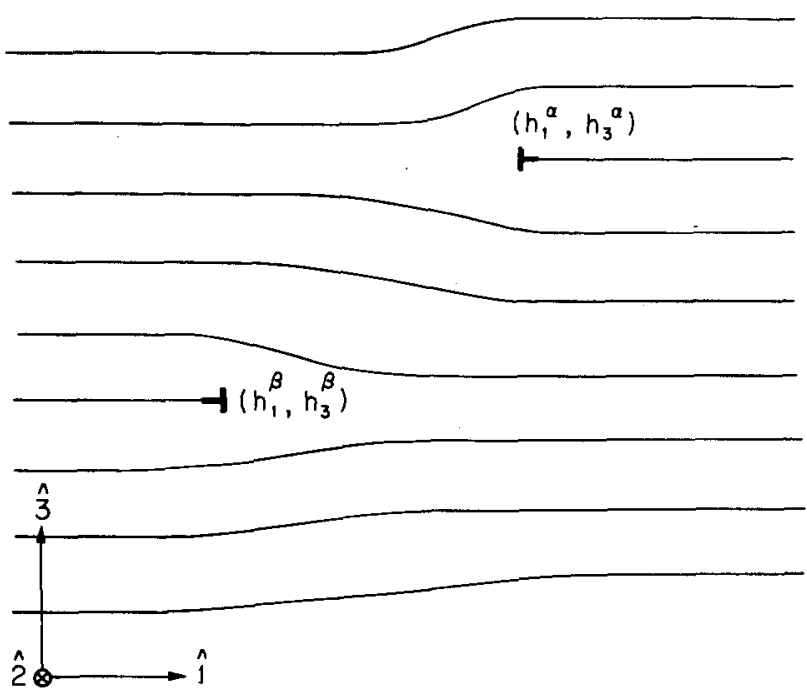

FIG. 2. A pair of edge dislocations $\alpha$ and $\beta$ at coordinate $\left(h \gamma, h_{3}\right), \gamma=\alpha, \beta$. The relative coordinates are $h_{1}=h_{1}^{\alpha}-h_{1}^{\beta}, h_{3}$ $=h_{3}^{\alpha}-h_{3}^{\beta}$ and the unit vector $\hat{2}$ is into the page.
Although for any plane ( $x_{3}=$ const), one can calculate the first moment of the $\hat{3}$ component of force per unit length of dislocation, that is the torque per unit length,

$$
\int_{-\infty}^{\infty} x_{1} \sigma_{33} d x_{1}=\frac{1}{2} \lambda a b,
$$

the results are not particularly useful. First, since the torque is independent of $x_{3}$, the distance between dislocation and surface, it will be sensitive to the presence of other dislocations. In Sec. IV we demonstrate that a variety of simple boundary conditions can be satisfied by the method of image dislocations. Observable torques thus depend on the boundary conditions.

\section{PAIRS OF DISLOCATIONS}

Consider a pair of dislocations at $\left(h_{1}^{\alpha}, h_{3}^{\alpha}\right)$ and $\left(h_{1}^{\beta}, h_{3}^{\beta}\right)$ as shown in Fig. 2. Neglecting the boundary effects in the same sense as in Sec. III, the components of strain field are the sum of the individual contributions from each dislocation

$$
\delta m_{i}\left(x_{1}, x_{3}\right)=\sum_{\gamma=\alpha, B} \delta m_{i}^{\gamma}\left(x_{1}, x_{3}\right),
$$

where $i=1,3$ and

$$
\begin{aligned}
\delta m_{1}^{\gamma}\left(x_{1}, x_{3}\right)= & -s_{\gamma}\left[4(\pi \lambda)^{1 / 2}\right]^{-1}\left(x_{3}-h_{3}^{\gamma}\right)\left|x_{3}-h_{3}^{\gamma}\right|^{-3 / 2} \\
& \times \exp \left[-\left(x_{1}-h_{1}^{\gamma}\right)^{2}\left(4 \lambda\left|x_{3}-h_{3}^{\gamma}\right|\right)^{-1}\right], \\
\delta m_{3}^{\gamma}\left(x_{1}, x_{3}\right)= & +\lambda\left(x_{3}-h_{3}^{\gamma}\right)\left|x_{3}-h_{3}^{\gamma}\right|^{-1} \partial_{1} \delta m_{1}^{\gamma}\left(x_{1}, x_{3}\right) .
\end{aligned}
$$

The quantity $s_{\gamma}$ specifies the sign of the dislocation. In the figure $s_{\alpha}=-s_{\beta}=+1$; however, what follows will be applicable to other cases.

There will be a force on dislocation $\alpha$ due to the stress field of dislocation $\beta, f_{i}^{\alpha, \beta}$. Strictly speaking, the force will depend on the boundary conditions; however, in the examples discussed here it will be possible to replace boundaries by image dislocations and calculate boundary forces in terms of the $f_{i}^{\alpha, \beta}$. Referring to Eq. (2.10), with $1^{\alpha}=-s_{\alpha} \hat{\mathbf{2}}$,

$$
\begin{aligned}
& f_{3}^{\alpha, \beta}=a s_{\alpha} \phi_{1}^{\beta}\left(h_{1}^{\alpha}, h_{3}^{\beta}\right), \\
& f_{1}^{\alpha, \beta}=-a s_{\alpha} \phi_{3}^{\beta}\left(h_{1}^{\alpha}, h_{3}^{\alpha}\right),
\end{aligned}
$$

where $\phi_{i}^{\beta}\left(h_{1}^{\alpha}, h_{3}^{\alpha}\right)$ is the stress field [Eq. (2.7)] at $\alpha$ due to $\beta$. If $h_{i}=h_{i}^{\alpha}-h_{i}^{\beta}$, the direct force of $\beta$ on $\alpha$ has components

$$
\begin{aligned}
f_{1}^{\alpha, \beta}= & \left(h_{1} /\left|h_{3}\right|\right)\left\{\frac{1}{8} s_{\alpha} s_{\beta} a^{2} b\left(\pi \lambda\left|h_{3}\right|\right)^{-1 / 2}\right. \\
& \left.\times \exp \left[-h_{1}^{2}\left(4 \lambda\left|h_{3}\right|\right)^{-1}\right]\right\}, \\
f_{3}^{\alpha, \beta}= & -\left(\lambda h_{3} /\left|h_{3}\right|^{2}\right)\left[h_{1}^{2}\left(2 \lambda\left|h_{3}\right|\right)^{-1}-1\right]\left\{\frac{1}{8} s_{\alpha} s_{\beta} a^{2} b\left(\pi \lambda\left|h_{3}\right|\right)^{-1 / 2}\right. \\
& \left.\times \exp \left[-h_{1}^{2}\left(4 \lambda\left|h_{3}\right|\right)^{-1}\right]\right\} .
\end{aligned}
$$

If $s_{\alpha} s_{\beta}>0$, that is, if both dislocations are of the same type, the direct force $f_{1}$ is repulsive; two dislocations of the same type will tend to displace themselves so as to avoid being directly over one another. Similarly, if $s_{\alpha} s_{\beta}<0$, the force is attractive and opposite dislocations would attract each other. The force $f_{3}$ is either attractive or repulsive depending on the relative positions of the two dislocations. Like dislocations $\left(s_{\alpha} s_{\beta}>0\right)$ will tend to increase their relative $h_{3}$ if $h_{1}^{2}<2 \lambda\left|h_{3}\right|$ and decrease it if $h_{1}^{2}>2 \lambda\left|h_{3}\right|$.

These effects are most easily visualized with the help of the potential 


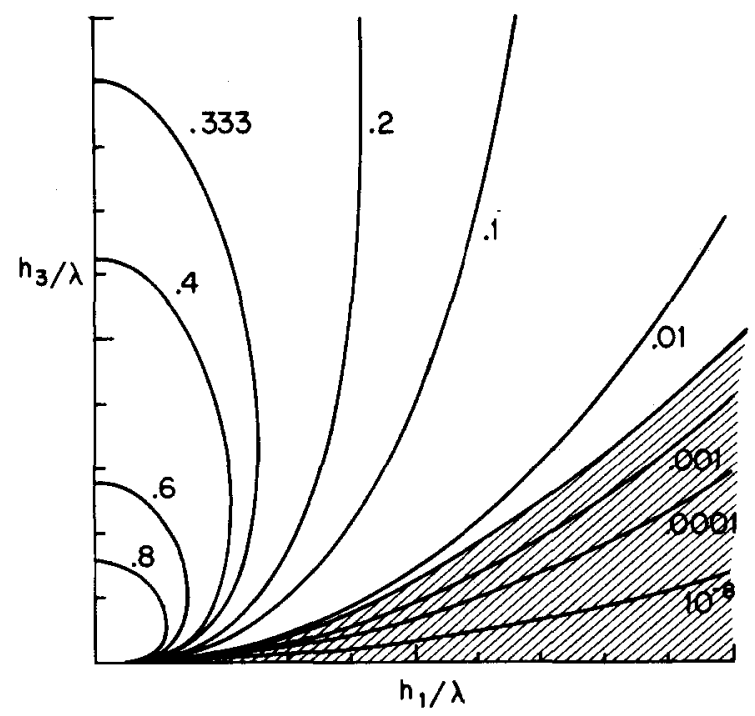

FIG. 3. Curves of constant energy of interaction as a function of relative posttion between two like edge dislocations $\left(s_{\alpha} s_{\beta}\right.$ $=+1)$. The energy parameter is given in units of $a^{2} b(4 \sqrt{\pi})^{-1}$. In these units $k T / a \sim 3.6 \times 10^{-3}$.

$\psi\left(h_{1}, h_{3}\right)=\frac{1}{4} s_{\alpha} s_{\beta} a^{2} b\left(\lambda / \pi\left|h_{3}\right|\right)^{1 / 2} \exp \left[-h_{1}^{2}\left(4 \lambda\left|h_{3}\right|\right)^{-1}\right]$.

The gradient of $\psi$,

$$
f_{i}=-\frac{\partial \psi}{\partial h_{i}}
$$

yields Eqs. (4.4). The potential $\psi$ is the excess smectic elastic energy per unit length of dislocation by virtue of the superposition of the strain fields of the two dislocations. ${ }^{27}$ In the Appendix we demonstrate this for a special case.

Figure 3 displays curves of constant $\psi$ as a function of reduced variables $h_{1} / \lambda$ and $h_{3} / \lambda$ for the example $s_{\alpha} s_{\beta}$ $=+1$. For typical values $b \approx 10^{10} \mathrm{erg} \mathrm{cm}^{-3},{ }^{28} a \approx 2 \times 10^{-7}$ $\mathrm{cm}$, the energy per $\mathrm{cm}$ of dislocation at a separation $\lambda$ along $h_{3}$ is $\Psi(0, \lambda) \approx 0.56 \times 10^{-4} \mathrm{erg} \mathrm{cm}^{-1}$. In order to evaluate the significance of this energy, $\Psi(0, \lambda) / T$ must be compared to the entropy per $\mathrm{cm}$ of dislocation. Nabarro estimates the entropy contribution to the free energy per atomic length of dislocation in a crystal as $\sim k T$. If we assume that this is approximately the correct value for the smectic, the entropy contribution to the free energy per unit length of dislocation is $k T / a \approx 2 \times 10^{-7}$ erg $\mathrm{cm}^{-1}$. The shaded region in Fig. 4.2 indicates where $k T / a \gtrsim \Psi$ and where thermal effects will dominate the elastic effects. ${ }^{29}$ The line separating the two regions will eventually curve over and meet the vertical axis similarly to the lines indicating larger potentials. The region in which elastic-interaction effects dominate is an elongated region along the $h_{3}$ axis. The width of the elongated region can roughly be defined by observing the curves of constant $\psi$ become vertical when $f_{3}^{\alpha, \beta}=0$. This defines a parabola

$$
\left|h_{3}\right|=(2 \lambda)^{-1} h_{1}^{2} \text {. }
$$

Inspection of Eq. (4.5) shows that for $\psi \approx k T / a,\left|h_{1}\right| / \lambda$ $\approx(2 e)^{-1 / 2}\left(a^{3} b / 4 k T\right) \sim 10^{+2}$. The "top" of the region is bounded by the condition $\psi\left(0, h_{3}\right) \approx k T / a$ or $\left(h_{3} / \lambda\right)$ $\approx\left(a^{3} b / 4 k T\right)^{2}(4 \pi e)^{-1} \sim 10^{4}$. If $\lambda$ is of the order of the layer spacing, the region dominated by elastic effects can be of the order of $10^{-3} \mathrm{~cm}$ along the vertical.

Although interaction between like dislocations is predominantly repulsive, two dislocations with relative positions that fall within the shaded region interact very weakly. Repulsive forces are such as to push like dislocations into the region of weak interaction. Strictly the line with $h_{3}=0$ is a line of zero potential and it is only because of thermal effects that the two dislocations do not take up just that position. This suggests that if external strains were to induce an excess of edge dislocations (see, for example, Fig. 3 of Ref. 7) they would tend to move to relative positions like those shown in Fig. 4 to form a "domain wall". This is identical to the model for polygonization of single crystals discussed by Nabarro. ${ }^{30}$ We will discuss this point at greater length below.

In the case of unlike dislocations $\left(s_{\alpha} s_{\beta}<-1\right)$ the potential changes sign and the relative position with $h_{3}=0$ is unstable. Although entropy effects dominate that region, one might expect that dislocations so placed will diffuse away from such a relative position (in the absence of pinning or other boundary forces) until they reach the region dominated by the elastic effects. Following that they will certainly attract and (unless dislocations have some type of short-range repulsive force) annihilate one another.

Boundary effects, however, can have significant effects on the stability or instability of different relative positions. These will be discussed below.

\section{BOUNDARY EFFECTS}

\section{A. Rigid surface}

Consider two dislocations, as shown in Fig. 2 except directly above one another $\left(h_{1}^{\alpha}=h_{1}^{\beta}\right)$. For simplicity take $h_{3}^{\alpha}=-h_{3}^{\beta}$. Equation (4.1) obtains that in the plane $x_{3}=0$ the strain $\delta m_{1}=\delta m_{1}^{\alpha}+\delta m_{1}^{\beta}=0$. From Eq. (2.4) one can see that in this configuration the plane $x_{3}=0$ coincides with a smectic layer. Conversely, if one assumes a semi-infinite smectic sample $\left(x_{3}<0\right)$ with a single dislocation a distance $h$ below the surface $\left(h_{3}=-h\right)$ and the boundary condition that the upper surface $\left(x_{3}=0\right)$ coin-

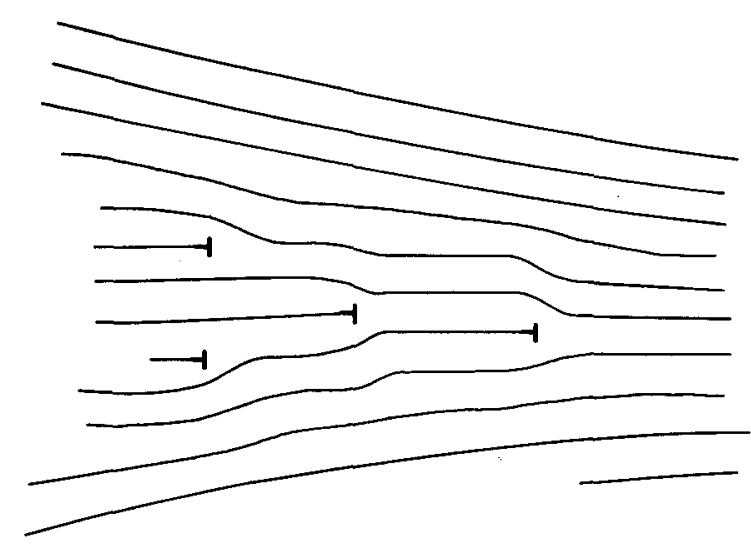

FIG. 4. Domain wall in smectic-A. 
cides with a smectic layer, the boundary value problem is solved by locating an image dislocation of the same type $\left(s_{\alpha} s_{\beta}=+1\right)$ directly above the true dislocation, a distance $h$ above the surface $\left(h_{3}=h\right)$. From Eq。(3.4) this surface also has $\sigma_{13}=0$.

The dislocation will experience a force per unit length (due to the image dislocation) repelling it from the surface

$$
f_{3}=-\frac{1}{16} a^{2} b(\lambda / 2 \pi)^{1 / 2}(h)^{-3 / 2} .
$$

The potential or elastic energy for the dislocation so located is

$$
\psi(h)=\frac{1}{8} a^{2} b(\lambda / 2 \pi)^{1 / 2} h^{-1 / 2} .
$$

This is just (2) $)^{-3 / 2}$ smaller than the energy associated with the potential $\psi(0, h)$ of Eq. (4.5) and illustrated in Fig. 3. Following the discussion pertaining to Fig. 3, the repulsive effect of the surface will dominate thermal effects up to distances $h \sim 10^{-3} \mathrm{~cm}$.

At the boundary plane $\left(x_{3}=0\right)$, the stress tensor $\sigma_{33}\left(x_{1}, 0\right)$ is

$$
\sigma_{33}=a b\left[4(\pi \lambda)^{1 / 2}\right]^{-1} x_{1} h^{-3 / 2} \exp \left(-x_{1}^{2} / 4 \lambda h\right) .
$$

Strictly speaking, this stress implies that there must be a torque per unit length on the boundary planes equal to $\int x_{1} \sigma_{33}(x) d x_{1}=\lambda a b \approx 4 \times 10^{-4}$ dyn $\mathrm{cm} / \mathrm{cm}$. Although such a torque is necessary if we rigidly adhere to the stated boundary conditions, the actual torque on the surface is sensitive to the number and distribution of dislocations in the sample and also to the slight deviations in the boundary conditions from the ideal.

\section{B. Free surface}

Two dislocations of opposite type directly above one another (Fig. 2 with $s_{\alpha} s_{\beta}=-1, h_{1}=0, h_{3}^{\alpha}=-h_{3}^{\beta}=h$ ) obtain $\sigma_{33}=a b \delta m_{3}=0$ in the plane $x_{3}=0$. Thus the solution for stress-strain fields of a single dislocation, a dis-

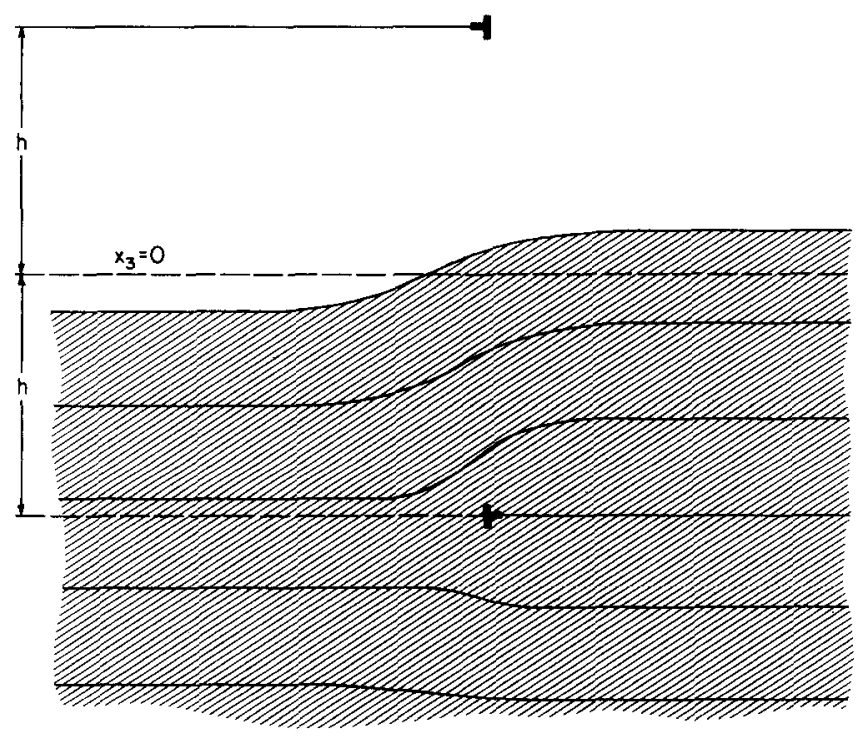

FIG. 5. Schematic illustration of the smectic layer configuration of $a+1$ dislocation a distance $h$ below a surface of zero stress $\sigma_{33}$. tance $h$ below a surface $\left(x_{3}=0\right)$ for which $\sigma_{33}=0$, is obtained by placing an "image dislocation" of opposite type a distance $h$ above the surface and directly over the true dislocation. Taking $s_{8}=-1$ in the boundary plane $\delta m_{1}$ $=-\left[2(\pi \lambda h)^{1 / 2}\right\}^{-1} \exp \left(-x_{1}^{2} / 4 \lambda h\right)$, corresponding to

$$
\partial_{1} \mu=a\left[2(\pi \lambda h)^{1 / 2}\right]^{-1} \exp \left(-x_{1}^{2} / 4 \lambda h\right) .
$$

Integration gives $\mu(\infty, 0)-\mu(-\infty, 0)=s_{\beta} a$. The smectic layer structure described here is shown in Fig. 5 for $s_{\beta}=-1$. The region far below the dislocation is relatively stress and strain free.

For this solution to be applicable, we must justify neglect of a surface tension contribution to the energy. The elastic energy per unit length of dislocation is attractive towards the surface with magnitude equal to $|\psi(h)|$ as given by Eq. (5.2). Surface tension effects are neglibible if this energy is large compared to the extra surface energy associated with the illustrated surface deformation. This requires the surface tension to be small compared to $b \lambda\left[2(2 \pi)^{1 / 2}\right]^{-1}$ which is of the order of $400 \mathrm{erg} \mathrm{cm}^{-2}$. Since surface tensions for organic liquids are rarely as large as $100 \mathrm{erg} \mathrm{cm}^{-2}$, surface tension effects are negligible.

Although an edge dislocation will be attracted towards a free surface, we are unable to discuss exactly what happens when the dislocation gets near the surface. Clearly there will be a step on the surface approximately one smectic layer high but the precise strain fields near the core and also in the bulk cannot be obtained from the elastic theory being presented here. The stress-strain field in the bulk can probably be described if one considers the surface step as equivalent to a pair of $s=+1$ and $s=-1$ dislocations at some relative spacing $\left(\delta h_{1}, \delta h_{3}\right)$; however, the sign and magnitude of this spacing would depend on specific properties of the surface tension, dislocation core, and the curvature of the dislocation. For example, suppose the dislocation forms a closed circular loop of radius $R$ on the surface corresponding to a disk of thickness $a$ sitting on top of the bulk smectic. Assuming a core energy per unit length $\gamma$ for the circumference of the disk $(\gamma>0)$, the energy of the smectic would be lowered if the disk could contract with the excess material in the disk diffusing into the bulk. If the disk does not contract, that would mean there was, in the bulk, some stress opposing molecular diffusion. Since the chemical potential for smectic liquid crystals has been introduced and expanded as a power series ${ }^{5}$ in the strain field, this problem can be treated in principle. It does seem, however, that the isothermal and isobaric assumptions of the present work are not sufficiently general. Realistic discussion of this type of problem is beyond the scope of the present manuscript.

\section{Stress-free surface $\left(x_{1}=\right.$ const $)$}

On inspection of Eqs. (3.3a) and (3.3b) or (3.4b) one can readily obtain image solutions to problems with boundaries at constant $x_{1}$ si bject to a variety of conditions. In all cases, however, the relative position of image to dislocation will always be at $h_{3}=0$ (see Fig. 2). From either Eq. (4.4) or Fig. 3, one can see there will be no direct interaction between dislocation and image. 


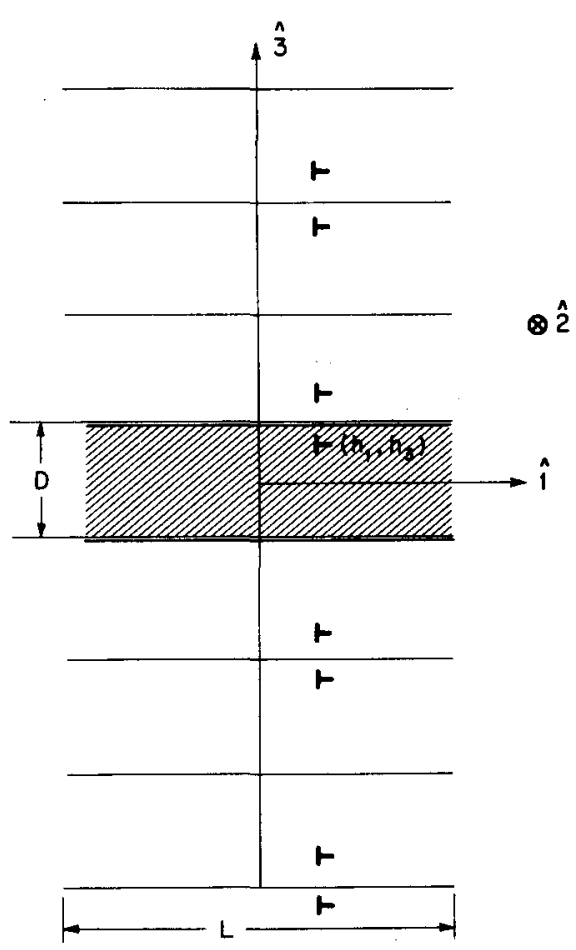

FIG. 6. Location of image dislocations for boundary conditions discussed in the text. The $\hat{2}$ direction is into the page. The sample is of dimension $L$ in the $\hat{1}$ direction and $\left|h_{1}\right| \ll L$.

We will see in subsequent sections that the horizontal forces on dislocations, towards or away from surfaces of constant $x_{1}$, are strongly influenced by the boundary conditions placed on the surfaces on constant $x_{3}$. For most practical purposes the dislocations can be assumed to be far from the boundaries at constant $x_{1}$.

Note, however, that these lateral boundaries are sources (and sinks) of dislocations. Stresses applied to the top and bottom surfaces can cause dislocations to move into (or out of) the sample through the lateral boundaries. See, for example, Secs. VIA and VIII below.

\section{SINGLE DISLOCATION IN A SMECTIC SAMPLE OF UNIFORM THICKNESS $D$}

\section{A. Rigid upper and lower boundaries}

Consider a dislocation $(s=+1)$ with coordinates $\left(h_{1} h_{3}\right)$ in a smectic- $A$ sample bounded at $x_{3}= \pm \frac{1}{2} D$ by surfaces of the type considered in Sec. VA $\left(\delta m_{1}=\sigma_{13}=\delta \mu=0\right)$. These boundary conditions are satisfied by an infinite set of image dislocations of the same sign $(s=+1)$ located at $\left(h_{1}, \pm n D+(-1)^{n} h_{3}\right), n=1,2,3, \ldots$. This is illustrated in Fig. 6. If we take the $\delta m_{i}$ and $\delta \sigma_{i j}$ given by Eqs. (3.3) and (3.4), respectively, to be the contribution of individual dislocations (real and image) to the total strain $\delta M_{i}$ and the total stress $S_{i j}$,

$$
\begin{aligned}
\delta M_{i}\left(x_{1}, x_{3}\right)= & \delta m_{i}\left(x_{1}-h_{1}, x_{3}-h_{3}\right) \\
& +\sum_{n}\left[\delta m_{i}\left(x_{1}-h_{1}, x_{3}-n D-(-1)^{n} h_{3}\right)\right. \\
& \left.+\delta m_{i}\left(x_{i}-h_{1}, x_{3}+n D-(-1)^{n} h_{3}\right)\right]
\end{aligned}
$$

$$
\begin{aligned}
S_{i j}\left(x_{1}, x_{3}\right)= & \sigma_{i j}\left(x_{1}-h_{1}, x_{3}-h_{3}\right) \\
& +\sum_{n}\left[\sigma_{i j}\left(x_{1}-h_{1}, x_{3}-n D-(-1)^{n} h_{3}\right)\right. \\
& \left.+\sigma_{i j}\left(x_{1}-h_{1}, x_{3}+n D-(-1)^{n} h_{3}\right)\right] .
\end{aligned}
$$

The force per unit length on the dislocation due to the boundary is obtained from Eq. $(2,10)$ with the identification

$$
\begin{aligned}
& \phi_{3}=-\left[S_{33}\left(h_{1}, h_{3}\right)-\sigma_{33}(0,0)\right], \\
& \phi_{1}=-\left[S_{13}\left(h_{1}, h_{3}\right)-\sigma_{13}(0,0)\right] .
\end{aligned}
$$

The force on the dislocation is due only to the stresses arising from the images; the stress of the dislocation itself must be subtracted.

Consider first the value of $S_{33}$ for $\left(x_{1}-h_{1}\right)^{2} / \lambda D \gg 1$. In the limiting case of this inequality, the summation over $n$ can be approximated as an integral:

$$
\begin{aligned}
S_{33}\left(x_{1}, x_{3}\right) \approx & a b(8 \sqrt{\pi} \lambda)^{-1}\left[2\left(x_{1}-h_{1}\right) / D\right] \\
& \times \int_{0}^{\infty} t^{-3 / 2} \exp \left[-\left(x_{1}-h_{1}\right)^{2}(4 \lambda t)^{-1}\right] d t .
\end{aligned}
$$

With the substitution $t=y^{-2}$, the integral is trivially evaluated:

$$
S_{33}\left(x_{1}, x_{3}\right) \rightarrow b(a / 2 D)\left(x_{1}-h_{1}\right)\left|x_{1}-h_{1}\right|^{-1} .
$$

This result can be obtained by a more intuitive argument that emphasizes the role of boundary conditions and external forces in determining the total stress strain fields. ${ }^{31}$ Since there is one more layer to the right of the dislocation than to the left, the strain $\delta m_{3}$ must change by an amount $\delta m_{3}=-\delta a / a^{2}=1 / D$ in passing the dislocation; $S_{33}\left(x_{1} \gg h_{1}\right)-S_{33}\left(x_{1} \ll h_{1}\right)=b a / D$. Assume that there are no external forces opposing $f_{1}$ and the dislocation is in some type of equilibrium state. This can only occur if the elastic energy does not change with small changes in $h_{1}$. From Eq. (2.6b) this will be true if $\left(\delta m_{3}\right)^{2}$ is the same for $x_{1} \gg h_{1}$ as for $x_{1} \ll h_{1}$. This implies $S_{33}\left(\infty, x_{3}\right)=-S_{33}\left(-\infty, x_{3}\right)$, which together with $S_{33}\left(\infty, x_{3}\right)-S_{33}\left(-\infty ; x_{3}\right)=b a / D$ gives Eq. $(6,3)$. From Eq. $(6.2 a)$ one can show directly that if the only stress fields are due to the images depicted in Fig. 6, the force per unit length $f_{1}=0$. From Eqs. (3.4) the individual contributions of each image to the summation (6.2a) vanish because the relative coordinate $x_{1}-h_{1}$ is zero; the images are directly above (or below) the dislocation.

This particular solution corresponds to the dislocation being in a state of neutral equilibrium with respect to $h_{1}$ motion. The elastic energy does not depend on the $h_{1}$ coordinate of the dislocation so long as the dislocation is far from the lateral boundaries at $x_{1}=$ const, i.e., so long as $\left(\frac{1}{2} L-\left|h_{1}\right|\right)^{2} \gg \lambda D$. This is obviously a rather special case since any slight change in the sample thickness $D$ (uniform or nonuniform) would alter the neutral equilibrium. We will later discuss the problem of a wedge-shaped sample in which $D$ increases linearly with position along the $x_{1}$ direction. Extra layers, implying dislocations, would presumably have to appear regularly wherever $D$ increases by one layer spacing. The wedge shape together with the interactions between dislocations and their images would presumably stabilize the pattern. For the moment, however, consider the case of a uniform change in sample thickness ( $\delta D$ independent of $x_{1}$ ). To be definite, consider superposing on the strain field 
of Eq. (6.1a) a uniform dilation $\delta m_{3}=-a^{-2} \delta a$ to obtain a total strain field

$$
\delta M_{3}^{*}=\delta M_{3}+\delta m_{3}
$$

From Eq。(3.4) the total stress

$$
S_{33}^{*}=S_{33}-b a^{-1} \delta a
$$

and $\phi_{3}\left(h_{1}, h_{3}\right)=b a^{-1} \delta a$ obtaining force $f_{1}=-b \delta a$. A slight increase in sample thickness $\delta D=-D a^{-1} \delta a$ produces a uniform force tending to move the dislocation through the sample, thereby relaxing the applied stress. In the absence of localized centers that might pin them, dislocations will always move in smectics in such a way as to exclude stress from the material. Even in the case of a perfect smectic with no dislocations, application of an external strain $\delta m_{3}$ could cause dislocations to move into the sample from the sides in order to reduce the net external force on the surface to zero. Constant stress $\left|S_{33}\right|>b a / D$ could induce a constant flow of dislocations through the sample boundaries. A key factor in analyzing this type of behavior is the relative probabilities for nucleating dislocation pairs (or loops) in the bulk relative to the surface. A practical consequence of the analogy between the smectic dislocation problem and the magnetic field problem (Sec. II) is that mobile dislocations cause smectics to have a "diaelasticity" that is similar to the diamagnetism of a material with mobile (free) electrons. Absolutely free dislocations would result in absolute diaelasticity analogous to superconductivity in the magnetic problem. ${ }^{32}$ Partially free dislocations would result in a macroscopic stress -strain relationship different from the intrinsic one defined by Eq. (3.4), with an effective $b^{\text {etf }}<b$. Thus any attempt to measure the intrinsic elastic constants of a smectic must always be done under conditions for which dislocation motion will not occur. That is, they must either be done at frequencies above some relaxation frequency for dislocation motion or under conditions where all dislocations are permanently pinned (if that is possible?). The pinning forces must be sufficient to balance the above force with negligible dislocation motions.

Assuming that the dislocation can somehow be stabilized against $\delta h_{1}$ motion, the boundary conditions alone can stabilize the dislocation against $\delta h_{3}$ motion. The force per unit length $f_{3}$ on a dislocation of the type shown in Fig。 6 is $f_{3}=a \phi_{1}$, where $\phi_{1}$ is given by Eq. $(6.2 b)$ :

$$
\begin{aligned}
f_{3}= & \lambda a^{2} b\left[8(\pi \lambda)^{1 / 2}\right]^{-1} \\
& \times \sum_{n=1,3,5}\left[\left(n D+2 h_{3}\right)^{-3 / 2}-\left(n D-2 h_{3}\right)^{-3 / 2}\right] .
\end{aligned}
$$

If $h_{3} \ll D$,

$$
f_{3} \approx-3 \lambda a^{2} b h_{3}\left[4(\pi \lambda)^{1 / 2}\right]^{-1} D^{-5 / 2} \sum_{l=0}(2 l+1)^{-5 / 2}
$$

and for a sufficiently thin sample the thermal fluctuations will be

$$
\left\langle\left(\frac{h_{3}}{D}\right)^{2}\right\rangle \approx \frac{4}{3}\left(\frac{\pi D}{\lambda}\right)^{1 / 2} k T\left(a^{3} b\right)^{-1} \frac{a}{\xi}\left(\sum_{l=0}(2 l+1)^{-5 / 2}\right)^{-1},
$$

where $\xi$ is the characteristic length along the dislocation. Typically, with $D \approx 1 \mu \mathrm{m}, \lambda \approx a$ gives $\left\langle\left(\delta h_{3} / D\right)^{2}\right\rangle$ $\leq 0.03(a / \xi)$. More generally, for larger $D$ the effects of the boundaries on localizing the dislocation can be esti- mated by integrating Eq. (6.6) to obtain the difference between the elastic energy with a dislocation at $h_{3}$ and one at $h_{3}=0$ :

$$
\begin{aligned}
E\left(h_{3}\right)-E(0)= & \lambda a^{2} b\left[8(\pi \lambda D)^{1 / 2}\right]^{-1} \sum_{n=1}^{\infty}\left[\left(2 n-1+\frac{2 h_{3}}{D}\right)^{-1 / 2}\right. \\
& \left.+\left(2 n-1-\frac{2 h_{3}}{D}\right)^{-1 / 2}-2(2 n-1)^{-1 / 2}\right]
\end{aligned}
$$

for $\left[\frac{1}{2} L-h_{1}\right]^{2} \gg \lambda D$. Figure 7 shows the curves of $E\left(h_{3}\right)$ $-E(0)$ as a function of $2 h_{3} / D$ in units of $k T / a$. (Values of $a, b, \lambda$, and $T$ are the same as were used throughout the paper.) Qualitatively the boundary effects are domi nated by the two image dislocations at $x_{3}= \pm D-h_{3}$. For $h_{3} \approx 0, E(0) \sim \lambda a^{2} b\left[8(\pi \lambda D)^{1 / 2}\right]^{-1}$ while for $h_{3} \approx \frac{1}{2} D, E\left(h_{3}\right)$ $\approx \lambda a^{2} b\left[8(\pi \lambda D)^{1 / 2}\right]^{-1}\left(1-2 h_{3} / D\right)^{-1 / 2}$. The potential barrier for the dislocation becomes comparable to $k T / \xi$ when

$$
[2 \Delta / D]^{-1 / 2}-1 \sim 8 k T(\pi D / \lambda)^{1 / 2}\left(a^{2} b \xi\right)^{-1},
$$

where $\Delta=\frac{1}{2} D-h_{3}$ is the distance between the dislocation and the nearest boundary. To obtain an upper limit for the fluctuations let $\xi \approx a$, for the typical numbers we have been assuming the right-hand side of $(6.8)$ is of the order of $7 \times 10^{-3}(D / \lambda)^{1 / 2}$. Thus for $D / \lambda \gtrsim 10^{6}$ the thickness of the barrier is greater than $\Delta / \lambda \sim\left(a^{3} b / 8 k T\right)^{2}(2 \pi)^{-1}$ which is roughly the same number as was discussed in Sec. IV, Fig. 3 in connection with a single boundary. On the other hand, for samples in which $D / \lambda \ll 10^{6}$ the bar rier becomes significant for $h_{3} / \lambda \approx\left(8 k T / a^{3} b\right) \pi^{1 / 2}(D / \lambda)^{3 / 2}$ $\sim 7 \times 10^{-3}(D / \lambda)^{3 / 2}$. For $D \approx 1 \mu \mathrm{m}, D / \lambda \approx 500$ and $h_{3} / D$ $\approx 0.15 \sim(0.04)^{1 / 2}$, which is approximately equal to the estimate resulting from Eq. $(6.8)$ with $\xi \approx a$. In thin smectic samples $(D \ll 1 \mu \mathrm{m})$ with rigid boundary conditions, dislocations will be largely confined to the central position, the exception being in the case where the surface is irregular.

de Gennes's ${ }^{13}$ initial discussion of the strain field of

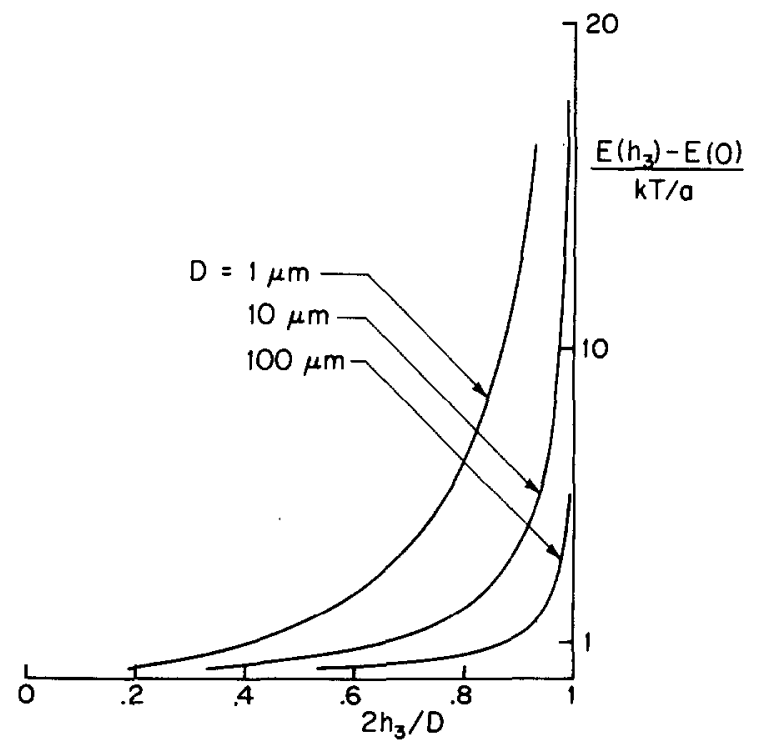

FIG. 7. Elastic energy per unit length as a function of $h_{3}$ for a dislocation located as shown in Fig. 6 and with boundary conditions as discussed in the text, $\left(\frac{1}{2} L-h_{1}\right)^{2} \gg \lambda D$. The energy is expressed in units of $k T / a$. See Eq. (6.9). 


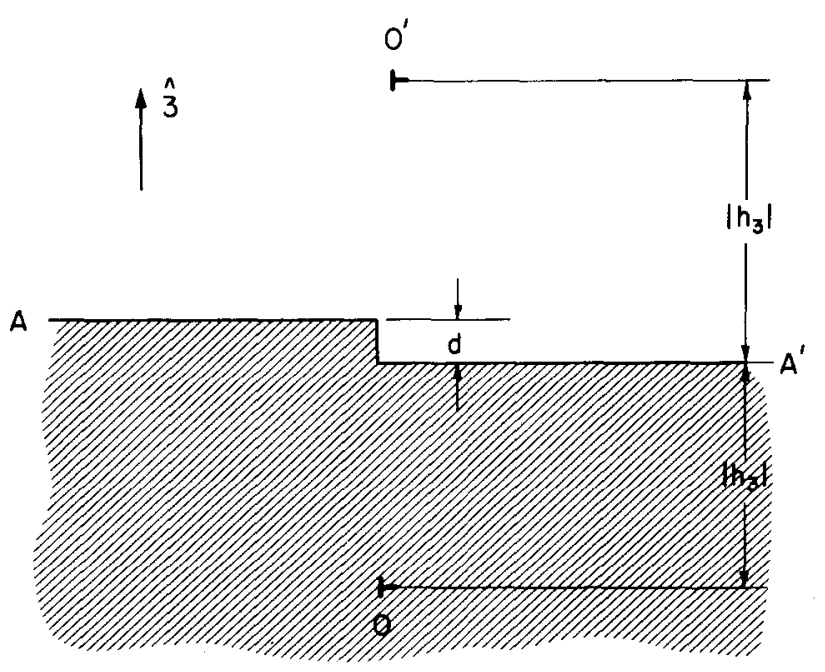

FIG. 8. A rigid boundary $\left(\mathrm{AA}^{\prime}\right)$ with a step of height $d$. The dislocation is at 0 and its image is at $0^{\prime}\left(h_{3} \gg d\right)$.

an edge dislocation emphasized that the same solution is applicable to a boundary that has a step of amplitude $\frac{1}{2} a$ (in our notation). In general, surface steps of any height $d$ are possible and the stress-strain field due to them should be the same as for the dislocation [Eqs . (3.3)] except that the amplitude should be scaled by a factor $2 d / a$. Figure 8 illustrates the positions of a surface step, a dislocation at 0 , and an image dislocation at $0^{\prime}$. We assume $h_{3} \gg d$, so that the exact position of $0^{\prime}$ is not critical. Neglecting the upper boundary, the net component of force $f_{3}$ on the dislocation is

$$
f_{3}=-\lambda a^{2} b\left[8(\pi \lambda)^{1 / 2}\right]^{-1}\left[\left(2 h_{3}\right)^{-3 / 2}-(2 d / a) h_{3}^{-3 / 2}\right] .
$$

If $2 d / a>2^{-3 / 2}$, the dislocation will be attracted to the step and, presumably pinned there. For a dislocation of opposite sign this step would be repulsive.

\section{B. Rigid lower boundary, upper boundary $\sigma_{33}=0$}

For a dislocation $(s=+1)$ located at shown in Fig. 9 with boundary conditions $\delta m_{1}=0$ on the lower surface and $\sigma_{33}=a b \delta m_{3}=0$ on the upper surface, the stressstrain solutions are obtained with dislocations at $h(n)$ $= \pm 2 n D+h$ of $\operatorname{sign} S_{n}=(-1)^{n} ; h^{\prime}(n)=(2 n-1) D-h$ with sign $S_{n}^{\prime}=(-1)^{n}$, and $h^{\prime \prime}(n)=-(2 n-1) D-h$ with sign $S_{n}^{\prime \prime}=(-1)^{n+1}$. Although it is straightforward to write down expressions for the stress -strain fields analogous to those of Sec. VIA, the most interesting quantity is the difference between the elastic energy per unit length for the dislocation located at $h_{3}$ and at $h_{3}=0$

$$
\begin{aligned}
E\left(h_{3}\right) & -E(0) \\
= & \lambda a^{2} b\left[8(\pi \lambda D)^{1 / 2}\right]^{-1} \\
& \times \sum_{n=1}^{\infty}(-1)^{n}\left[\left(2 n-1+\frac{2 h}{D}\right)^{-1 / 2}-\left(2 n-1-\frac{2 h}{D}\right)^{-1 / 2}\right] .
\end{aligned}
$$

Equation (6.12) is plotted versus $2 h / D$ in units of $k T / a$ in Fig. 10. The elastic energy becomes increasingly negative as the dislocation moves towards the upper surface $(2 h / D=+1)$. The figure illustrates that for a smectic drop on a glass plate (with rigid boundary conditions) and a free upper surface (with boundary conditions $\sigma_{33}=0$ ) dislocations will be expelled towards the free surface. For thin samples $(D \leqslant 1 \mu \mathrm{m})$ the potential gradient is large and the expulsion should be rapid. For thicker samples $(D \geq 100 \mu \mathrm{m})$ the potential gradient is large only near the boundaries and there is an extensive region is which dislocation motions may be dominated by other effects. ${ }^{29}$

In this example, as in the previous example, the boundary effects are qualtitatively dominated by the nearest image as the dislocation approaches a surface. The numerical estimates that were made in Sec. VIA on the dimensions of the regions in which the boundary potentials are significant are applicable here also.

\section{Upper and lower boundaries with $\sigma_{33}=0$}

A free smectic film of the type discussed by Clark and Meyer ${ }^{33}$ might be described in terms of the geometry being discussed here with boundary conditions on both the upper and lower surfaces of $\sigma_{33}=0$. With these boundary conditions and a dislocation located at $(0, h)$, the image solution is obtained by placing image dislocations at $h_{3}(n)= \pm n D+(-1)^{n} h$ with sign $S_{n}=(-1)^{n}$. The elastic energy is given by

$$
\begin{aligned}
E\left(h_{3}\right)- & E(0) \\
= & \lambda a^{2} b\left[8(\pi \lambda D)^{1 / 2}\right]^{-1} \times \sum_{n=1}^{\infty}(-1)^{n}\left[\left(2 n-1+\frac{2 h}{D}\right)^{-1 / 2}\right. \\
& \left.+\left(2 n-1-\frac{2 h}{D}\right)^{-1 / 2}-2(2 n-1)^{-1 / 2}\right] .
\end{aligned}
$$

Equation (6.13) is plotted in Fig. 11. The curves are almost identical to those of Fig. 7 except for being in-

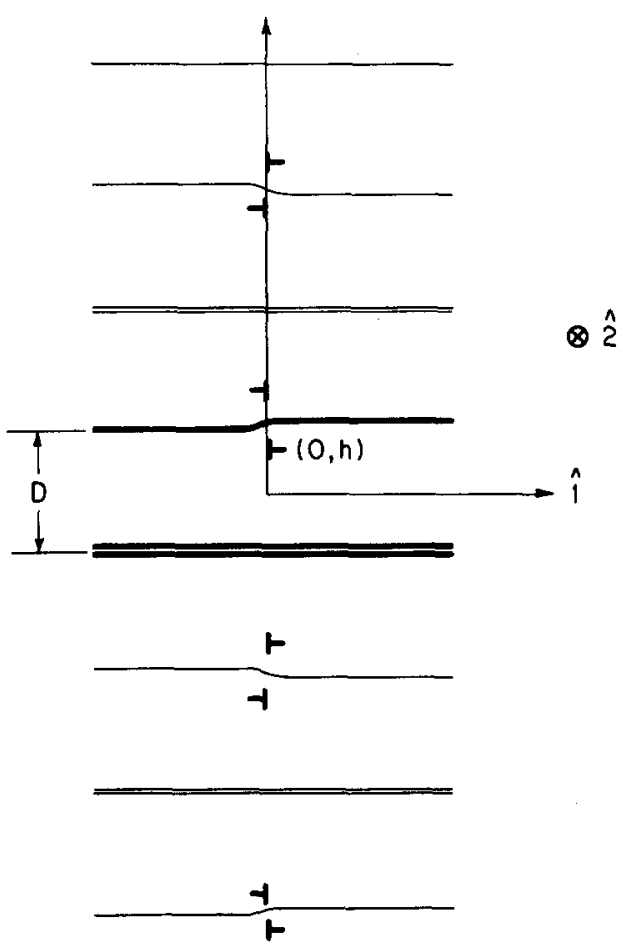

FIG. 9. Dislocation at $\left(0, h_{3}\right)$ with boundaries $x_{3}= \pm \frac{1}{2} D$ of the type described in the text. Image positions and signs are indicated. 
verted. Edge dislocations in this type of sample will be unstable and move to either surface. Effects dependent on sample thickness follow the same type of arguments given for the previous examples and the same numerical estimates apply. For practical purposes once dislocations get near the surface the comments in Sec. VC apply.

\section{WEDGE-SHAPED SAMPLE}

\section{A. Thick samples}

The wedge-shaped sample mentioned in Sec. VIA appears to be practical for the study of dislocations. Assuming rigid boundary conditions, if one starts from a flat sample that is relatively free from defects ${ }^{28}$ and squeezes one end so as to wedge the sample, edge dislocations would probably form. Assuming a wedge angle $\theta \ll \pi$, the number of dislocations per unit distance that are required to minimize the total strain energy is $d^{-1}$ $=a^{-1} \theta$, where $d$ is the average distance between dislocations. ${ }^{7}$ The minimum elastic energy results from locating the dislocations at $x_{1}=n d+\frac{1}{2} d$, where the origin $\left(x_{1}=0\right)$ is the vertex of the wedge angle and $n=n^{0}, n^{0}$ $+1, n^{0}+2 \ldots$; the $x_{1}$ coordinate of the first dislocation is $x_{1}^{0}=\left(n^{0}+\frac{1}{2}\right) d$. Ignoring the boundaries for the moment, the direct contribution of the dislocations to the strain $\delta M_{1}^{D}$ is

$$
\begin{aligned}
\delta M_{1}^{D}= & -\left[4(\pi \lambda)^{1 / 2}\right]^{-1} x_{3}\left|x_{3}\right|^{-3 / 2} \\
& \times \sum_{n=n^{0}}^{\infty} \exp \left[-\left(x_{1}-\frac{1}{2} d-n d\right)^{2}\left(4 \lambda\left|x_{3}\right|\right)^{-1}\right] .
\end{aligned}
$$

If $d^{2} \ll \lambda\left|x_{3}\right|, E q .(7.1)$ can be approximated by an integral

$$
\begin{aligned}
\delta M_{1}^{D} \approx & -\left[4(\pi \lambda)^{1 / 2}\right]^{-1} x_{3}\left|x_{3}\right|^{-3 / 2} d^{-1} \\
& \times \int_{-\left(x_{1}-x_{1}^{0}\right)}^{\infty} \exp \left[-\left(s^{2} / 4 \lambda\left|x_{3}\right|\right)\right] d s \\
\approx & -(4 d)^{-1}\left(x_{3} /\left|x_{3}\right|\right)\left[1+\operatorname{erf}\left[\left(x_{1}-x_{1}^{0}\right)\left(4 \lambda\left|x_{3}\right|\right)^{-1 / 2}\right]\right],
\end{aligned}
$$

where erf is the error integral. For thick enough samples the inequality is satisfied near the boundaries. Far from the sample edge, where $x_{1}-x_{1}^{0} \gg\left(2 \lambda\left|x_{3}\right|\right)^{1 / 2}$,

$$
\delta M_{1}^{D} \approx-(2 d)^{-1} x_{3}\left|x_{3}\right|^{-1}
$$

and [from $E q .(3,3 b)]$

$$
\delta M_{3}^{D} \approx 0 \text {. }
$$

On integration of $\partial_{1} \mu=-a \delta M_{1}^{D}$ the layer displacement associated with $(7.3)$ is

$$
\mu\left(x_{1}, x_{3}\right)=(a / 2 d)\left(x_{3}\left|x_{3}\right|^{-1}\right) x_{1} \text { 。 }
$$

Thus for a sufficiently thick sample the far-field pattern of the strain field [Eq. (7.1)] is compatible with rigid wedged boundaries. In this sense $\mathrm{Eq} .(7.1)$ is an asymptotic solution to the wedge problem. Note that in addition to satisfying the wedge boundary conditions the $\delta M_{1}, \delta M_{3}$ of Eqs. $(7.3)$ also satisfy the homogeneous differential equations [Eqs. (3.1)] for the strain field away from the dislocations.

Equations (7.3) fail as an exact solution to the wedge problem because they do not describe the strain field near the dislocations. Equation (7.1) describes this region satisfactorily but the layer displacements obtained from it are not perfectly flat near the boundaries. This latter fault is the easier of the two to improve upon.

In Sec. VA we saw that images placed symmetrically about a rigid boundary, properly oriented, can exactly cancel the nonuniform layer displacements at the boundary. We also saw in Sec. VIA that although samples of finite thickness require an infinite series of image dislocations, the size of the energy barrier near the surface was dominated by the images nearest to the surface. On the other hand, the stabilizing forces against motion in the $x_{1}$ direction were determined by the local stress $\sigma_{33}=-\phi_{3}$ which depends on only the sample thickness and the number of smectic layers in that thickness. Thus for a number of purposes one might consider as an approximate solution to the wedge problem the superposition of Eq. (7.1) and only the first set of image dislocations on the far side of the two boundaries. Figure 12 illustrates this approximation. Taking the equilibrium positions for the dislocations $x_{1}(n)=\left(\frac{1}{2}+n\right) d, x_{3}=0$ with $d^{-1}=a^{-1} \theta$ ensures local values of $\sigma_{33}$ such that the force $f_{1}$ on individual dislocations is zero. Image dislocations at $x_{1}(n)=\left(\frac{1}{2}+n\right) d$ and $x_{3}= \pm D\left(x_{1}\right)$, with $D\left(x_{1}\right)=\theta x_{1}(n)$ smooth out most of the nonuniformity in the $\delta M_{1}^{D}$ described by Eq. (7.1). Although there will be a small residual $f_{1}$ on each dislocation because the images on either side have $x_{3}$ coordinates $D\left[x_{1}(n+1)\right]>D\left[x_{1}(n-1)\right]$, the effects are not important for thick samples.

This image distribution can be interpreted either as an approximate solution to the wedge problem or an exact solution to a wedge sample in which the boundaries are not perfectly flat but ripple slightly to accommodate the small residual $\delta M_{1}$ at the boundaries. The average value of $\delta M_{1}$ is $(2 a)^{-1} \theta$ and the residual ripple in $\delta M_{1}$ is of the order of $(D a)^{-1 / 2}$. If $D a \gg(a / \theta)^{2}$ $=d^{2}$, the ripple is small. Dislocation densities satisfy ing this inequality will be referred to as low density. In this case the solutions of Sec. VIA are approximately applicable and the dominant contribution to $\sigma_{13}$ $=-\phi_{1}[\mathrm{Eq} .(3.4)]$ at the site of a particular dislocation arises from the dislocation's own first image. Further, that force is only large when the dislocation approaches the surface (Fig。7). The images stabilize the dislocation against vertical motion exactly as in Sec.VIA.

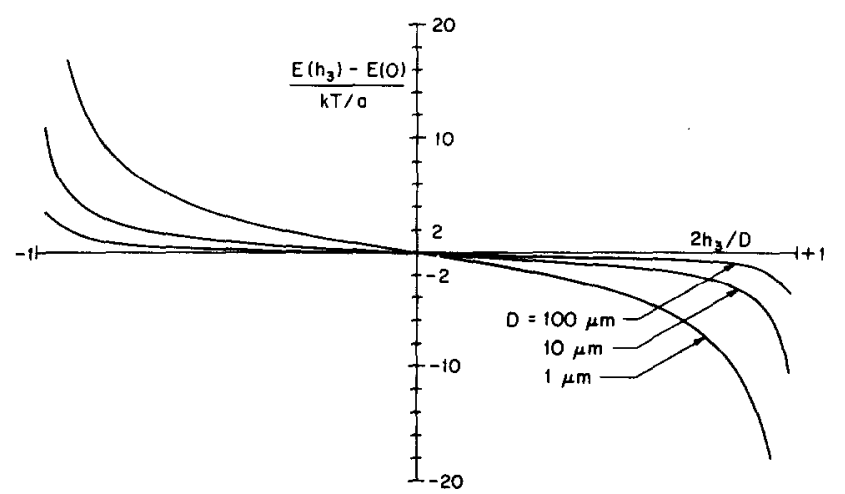

FIG. 10. Elastic energy per unit length as a function of $h_{3}$ for a dislocation located as in Fig. 9. See Eq. (6.12). 


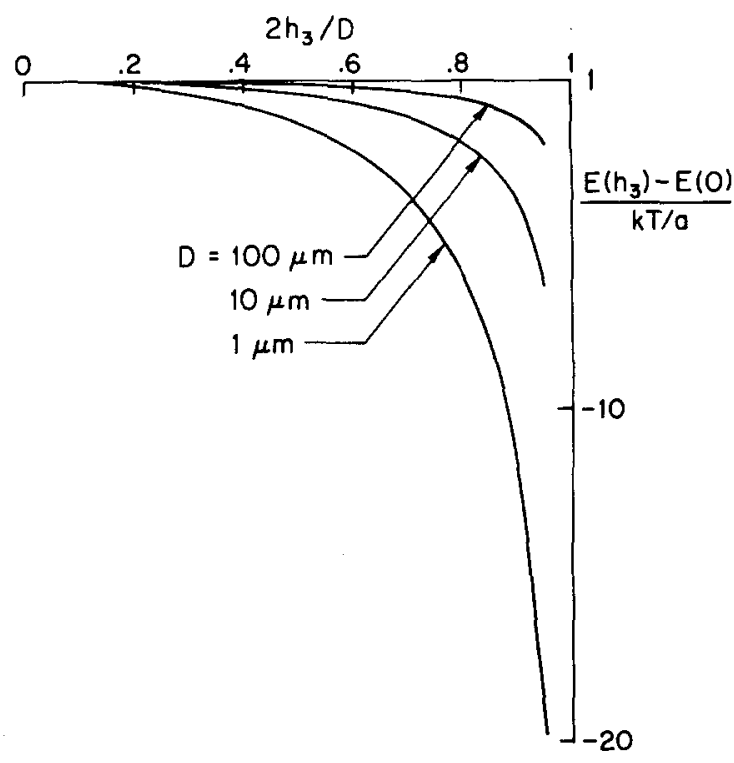

FIG. 11. Elastic energy per unit length as a function of $h_{3}$ for the problem discussed in Sec. VIC; see Eq. (6.13).

The force $f_{1}$ on a dislocation is determined by Eq. $(2.10), f_{1}=-a \phi_{3}$, where $-\phi_{3}$ is the average of the $\sigma_{33}$ to the right and to the left of the dislocation. Assuming some equilibrium value of $x_{1}(n)$, a dislocation moving to the right by $\delta x_{1}$ increases the average strain $\delta a / a$ by $\theta \delta x_{1} / D\left(x_{1}\right)$ and since $\phi_{3}=-a b \delta m_{3}=b \delta a / a=b \theta D\left[x_{1}(n)\right]^{-1}$ $\times \delta x_{1}$, the restoring force is

$$
f_{1}=-a b \theta D\left(x_{1}\right)^{-1} \delta x_{1} .
$$

The dislocation will be stabilized at some position $x_{1}(n)$ if

$$
\left\langle\left(\delta x_{1} / d\right)^{2}\right\rangle=k T D\left(x_{1}\right) \theta\left(a^{4} b\right)^{-1}(a / \xi) \ll 1 .
$$

For typical numerical values

$$
\left\langle\left(\delta x_{1} / d\right)^{2}\right\rangle \approx 2.5 \times 10^{3} D\left(x_{1}\right) \theta(a / \xi) .
$$

Taking $D\left(x_{1}\right) \sim 1 \mu \mathrm{m}$ and $\theta \sim 0.1^{\circ}$ we obtain $\left\langle\left(\delta x_{1} / d^{2}\right)\right\rangle$ $\sim 4 \times 10^{-4} a / \xi$ with $d \sim 10^{-4} \mathrm{~cm}$. Although wedges with larger angles would have larger restoring forces [Eq. (7.5)], they would also have larger dislocation densities, i.e., smaller $d$. The net effect is an increase in the relative fluctuations with decreasing $d$.

de Gennes ${ }^{13}$ estimated the optical cross section for scattering by the strain pattern surrounding an individual dislocation pinned to one boundary in terms of the Fourier transform of the optical inhomogeneity induced by $\delta m_{1}$. The result is readily extended to both real or image dislocations located near flat boundaries. The scattering amplitude from an array of dislocations is just a linear combination of these individual amplitudes. Although the location of the boundaries relative to the dislocation effects the precise dependence of the scattering amplitude on scattering wave vector $\mathrm{K}=\mathrm{k}^{\mathrm{scat}}-\mathrm{k}^{\mathrm{inc}}$, the scattering intensity is largely confined to the region of $(\mathrm{K})_{3} \approx 0.10,11,34$

For small $d$, that is in the high-density region with $d^{2} \leq D a$, the approximation under discussion is inadequate and we have not been able to construct an acceptable solution. Even if we found some way to satisfy the boundary conditions, dislocations packed this densely will interact strongly with each other. The problem then becomes a many-body one and the correct choice for the configuration of minimum energy is not obvious. For example, one possibility is that as $\theta$ increases, causing the dislocation density $d^{-1}=a^{-1} \theta$ to exceed $(D a)^{-1 / 2}$, the single dislocation wall splits into two, each of which has density $(2 a)^{-1} \theta$. Further increase in $\theta$ might further proliferate the number of walls while essentially keeping the horizontal spacing between dislocations of the order of $(D a)^{-1 / 2}$. Delaye, Ribotta, and Durand ${ }^{34}$ observed light-scattering effects in smectic samples under conditions where wedgelike behavior is possible. Although they interpreted their data by a different (although perfectly plausible) explanation, their principal observations were that by touching the sample on one edge they established a "periodic deformation" with spacing that is essentially given by $d^{2} \sim D a$ [see Eq. (3) of Ref. 34]. Although there are ambiguities as to factors of $\pi$ (i.e., $\lambda=a$ or $\lambda=a / 2 \pi$ ), the order of magnitude of the low-density criterion is that for a $100-\mu \mathrm{m}$ thick sample $\theta \leqslant 2^{\circ}$.

\section{DISLOCATION LOOP}

Consider a circular dislocation loop of radius $R$ in the plane of the smectic layers. Using a right-handed set of cylindrical coordinates $\left(\rho, \theta, x_{3}\right)$ we assume the dislocation density appropriate to the loop is

$$
1=-\hat{\theta} \delta(\rho-R) \delta\left(x_{3}\right),
$$

where the sign of the loop is such that the extra layer is exterior to the loop. If the Fourier transform of the Cartesian components of (8.1) are expressed in terms of cylindrical coordinates appropriate to reciprocal space:

$$
\begin{aligned}
& l_{i}\left(q, \phi, q_{3}\right)= \int l_{i}\left(\rho, \theta, x_{3}\right) \exp \left\{-i\left[q \rho \cos (\theta-\phi)+q_{3} x_{3}\right]\right\} \\
& \times \rho \rho \rho d \theta d x_{3} \\
& l_{1}(q)=-2 \pi i R \sin \phi J_{1}(q R) \\
& l_{2}(q)=+2 \pi i R \cos \phi J_{1}(q R) \\
& l_{3}(q)=0
\end{aligned}
$$

where $J_{1}$ is the Bessel function of first order. The same methods as were used in Sec. III can be employed here:

$$
\begin{aligned}
& i[\mathrm{q} x \delta \mathrm{m}(q)]=1(q), \\
& q_{3} \delta m_{3}(q)+\lambda^{2} q^{3}\left[\delta m_{1}(q) \cos \phi+\delta m_{2}(q) \sin \phi\right]=0 .
\end{aligned}
$$

From Eqs. (8.3) and (8.4)

$$
\begin{aligned}
& \delta m_{3}(q)=-2 \pi \lambda^{2} R q^{3} J_{1}(q R)\left(q_{3}^{2}+\lambda^{2} q^{4}\right)^{-1} \\
& \delta m_{3}\left(\rho, \theta, x_{3}\right)=-(2 \pi)^{-2} \lambda^{2} R \\
& \quad \times \int J_{1}(q R)\left(q_{3}^{2}+\lambda^{2} q^{4}\right)^{-1} \exp \left\{i\left[q \rho \cos (\theta-\phi)+q_{3} x_{3}\right]\right\} \\
& \quad \times q^{4} d q d q_{3} d \phi .
\end{aligned}
$$

The integrals over $q_{3}$ and $\phi$ are straightforward;

$$
\delta m_{3}=-\frac{1}{2} \lambda R \int_{0}^{\infty} J_{0}(q \rho) J_{1}(q R) \exp \left(-\lambda q^{2}\left|x_{3}\right|\right) q^{2} d q .
$$


Since $J_{1}(q R)=-q^{-1} \partial J_{0}(q R) / \partial R$,

$$
\delta m_{3}=+\frac{R}{2} \frac{\partial}{\partial R} \int_{0}^{\infty} J_{0}(q \rho) J_{0}(q R) \exp \left[-\lambda q^{2}\left|x_{3}\right|\right] q d q
$$

which is equal to ${ }^{35}$

$$
\begin{aligned}
\delta m_{3}= & +R\left(4\left|x_{3}\right|\right)^{-1} \frac{\partial}{\partial R}\left\{\exp \left[-\left(\rho^{2}+R^{2}\right)\left(4 \lambda\left|x_{3}\right|\right)^{-1}\right]\right. \\
& \left.\times I_{0}\left[\rho R\left(2 \lambda\left|x_{3}\right|\right)^{-1}\right]\right\},
\end{aligned}
$$

where $I_{0}(z)$ is the modified Bessel function of zero order:

$$
I_{0}(z)=\sum_{m=0}^{\infty}\left(\frac{z}{2}\right)^{2 m}[m !]^{-2}=J_{0}(i z) .
$$

In the limit that $\rho R \gg 2 \lambda\left|x_{3}\right|$, the asymptotic form for

$$
I_{0}(z) \rightarrow(2 \pi z)^{-1 / 2} \exp (+z)
$$

gives, as $\left|x_{3}\right| \rightarrow 0$,

$$
\begin{aligned}
\delta m_{3} \rightarrow & {\left[8(\pi \lambda)^{1 / 2}\right]^{-1}(R / \rho)^{1 / 2}(\rho-R)\left|x_{3}\right|^{-3 / 2} } \\
& \times \exp \left[-(R-\rho)^{2}\left(4 \lambda\left|x_{3}\right|\right)^{-1}\right] .
\end{aligned}
$$

For $R \approx \rho$ and $\left|x_{3}\right| \ll R^{2} / \lambda$ this is exactly equal to the result obtained in Sec. III for the strain field $\delta m_{3}\left(x_{1}, x_{3}\right)$ with $x_{1}=\rho-R$ [see Eqs. (3.3)].

To obtain the strain field $\delta m_{\rho}\left(\rho, \theta, \tilde{x}_{3}\right)$, observe that away from the dislocation

$$
\frac{\partial m_{\rho}}{\partial x_{3}}=\frac{\partial m_{3}}{\partial \rho} \text {. }
$$

From Eq. (8.8) and the properties of Bessel functions

$$
\begin{aligned}
\frac{\partial}{\partial x_{3}}\left(\rho^{-1} \frac{\partial\left(\rho m_{\rho}\right)}{\partial \rho}\right)= & -\frac{R}{2} \frac{\partial}{\partial R} \int_{0}^{\infty} J_{0}(q \rho) J_{0}(q R) \\
& \times \exp \left(-\lambda q^{2}\left|x_{3}\right|\right) q^{3} d q,
\end{aligned}
$$

and for $x_{3} \neq 0$

$$
\begin{aligned}
\rho^{-1} \frac{\partial\left(\rho m_{\rho}\right)}{\partial \rho}= & +x_{3}\left|x_{3}\right|^{-1} R(2 \lambda)^{-1} \\
& \times \frac{\partial}{\partial R} \int_{0}^{\infty} J_{0}(q \rho) J_{0}(q R) \exp \left(-\lambda q^{2}\left|x_{3}\right|\right) q d q .
\end{aligned}
$$

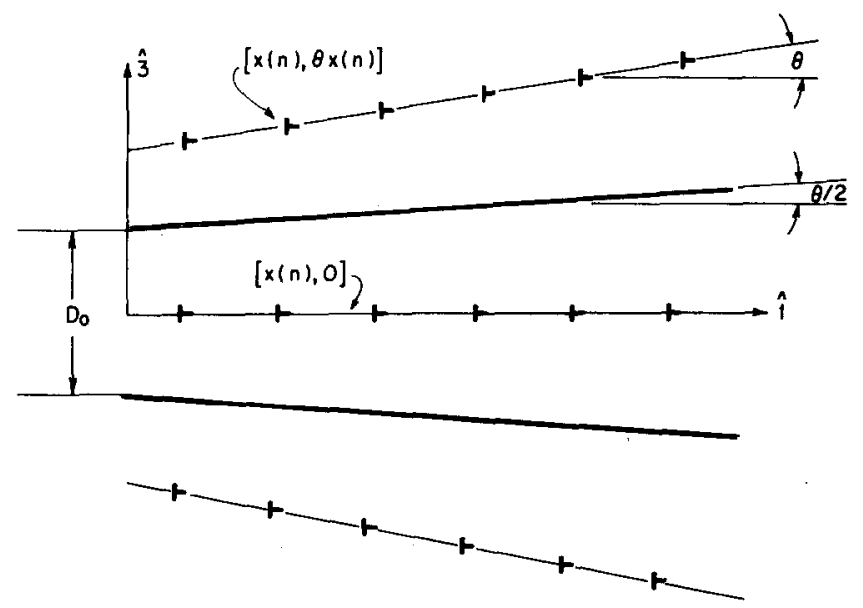

FIG, 12. An image distribution to approximate the solution to the wedge problem discussed in the text.
In place of Eq. (3.3b) we have

$$
\delta m_{3}=\lambda x_{3}\left|x_{3}\right|^{-1}\left(\rho^{-1} \frac{\partial\left(\rho m_{\rho}\right)}{\partial \rho}\right) \text {. }
$$

Light-scattering cross sections for dislocation loops can be estimaged by de Gennes's method ${ }^{13}$ using the identities

$$
\delta m_{1}(q)=\left[q m_{3}(q) \cos \phi-i l_{2}(q)\right] q_{3}^{-1}
$$

or

$$
\delta m_{1}(q)=2 \pi R \cos \phi q_{3} J_{1}(q R)\left(q_{3}^{2}+\lambda^{2} q^{4}\right)^{-1}
$$

and similarly

$$
\delta m_{2}(q)=2 \pi R \sin \phi q_{3} J_{1}(q R)\left(q_{3}^{2}+\lambda^{2} q^{4}\right)^{-1} .
$$

The strain fields of a dislocation loop midway between the type of flat rigid boundaries shown in Fig. 6 will be given by an infinite set of image dislocation loops directly over (and under) the original at $x_{3}=n D$. At any point $\left(\rho, \theta, x_{3}\right)$ the stress $\sigma_{33}$ will be the sum

$$
\sigma_{33}=a b \sum_{n=-\infty}^{\infty} \delta m_{3}\left(\rho, x_{3}-n D\right),
$$

where $\delta m_{3}$ is given by either Eq. (8.7), (8.8), or (8.9) using Eq. $(8.7)$ at $x_{3}=0$ :

$$
\sigma_{33}=-\frac{1}{2} a b \lambda R \int_{0}^{\infty} J_{0}(q \rho) J_{1}(q R) \operatorname{coth}\left(\lambda q^{2} D / 2\right) q^{2} d q .
$$

For thin samples, $(\lambda D)^{1 / 2} \ll R$, this is approximately

$$
\sigma_{33} \approx-a b R D^{-1} \int_{0}^{\infty} J_{0}(q \rho) J_{1}(q R) d q,
$$

which can be integrated to obtain ${ }^{36}$

$$
\begin{aligned}
\sigma_{33} & =-a b / D & & \text { if } 0<\rho<R, \\
& =0 & & \text { if } R<\rho<\infty .
\end{aligned}
$$

With perfectly flat boundaries and the implicit assumption that the region exterior to the loop is stain free, Eq. $(8.18)$ is the obvious result. The interior has one less layer than the exterior and has a strain $\delta a / a=a / D$.

Equation (8.18) predicts the contribution of the dislocation and boundaries to the stress without regard to the forces maintaining the loop. Similar to the problems raised earlier with regard to the straight dislocation, it will be necessary to superpose on the stress-strain fields of Eqs. (8.15)-(8.18) external fields sufficient to maintain the loop in equilibrium. In contrast to the straight dislocation, for the loop we must also consider the energy per unit length associated with the dislocation core. This can be expressed as a line tension $\gamma$ with dimensions of erg $\mathrm{cm}^{-1}$. The core energy cannot be calculated from the macroscopic elastic theory and we introduce it here as a phenomenological parameter, $\gamma>0$. The ratio $\gamma / b$ has dimensions of length squared and a reasonable guess would be

$$
\gamma / b \approx a^{2} .
$$

The combination of line tension and stress induces a force per unit length on the dislocation,

$$
f_{\rho}=-a \phi_{3}-\gamma R^{-1} \text {, }
$$

where $\phi_{3}=-2^{-1}\left[\sigma_{33}(\rho>R)+\sigma_{33}(\rho<R)\right]$. In the absence of inhomogeneities that might contribute further forces, 
the condition $f_{p}=0$ implies a state of unstable equilibri um for uniform boundary conditions. For example, under conditions of constant thickness $\sigma_{33}(\rho>R)$, $\sigma_{33}(\rho<R)$, and $\phi_{3}$ are constant, independent of $R$. If $\phi_{3}$ $=-\left(a R_{1}\right)^{-1} \gamma$ is the equilibrium stress at some radius $R_{1}$,

$$
\left.\frac{\partial f_{\rho}}{\partial R}\right|_{R=R_{1}}=\frac{\gamma}{R_{1}^{2}}>0,
$$

implying instability 。

Similarly the dislocation is in unstable equilibrium under conditions of constant external force $F_{3}$ applied to the upper boundary,

$F_{3}=-\pi R_{0}^{2} \sigma_{33}(\rho>R)-\pi R^{2}\left[\sigma_{33}(\rho<R)-\sigma_{33}(\rho>R)\right]$,

where $\pi R_{0}^{2}$ is the area of the flat surfaces. From Eq. $(8.18)$ this can also be written

$$
F_{3}=-\pi R_{0}^{2} \sigma_{33}(\rho>R)+\pi R^{2} a b D^{-1}
$$

From Eqs. (8.18) and (8.23), Eq. (8.20) can be written

$$
f_{\rho}=-a\left(\pi R_{0}^{2}\right)^{-1} F_{3}+a^{2} b D^{-1}\left[\left(R / R_{0}\right)^{2}-\frac{1}{2}\right]-\gamma R^{-1}
$$

and $\partial\left(f_{\rho}\right) / \partial R>0$.

Consider, however, the case of a perfect smectic sample, free of any dislocations, and with initial thickness $D$ that is an integral multiple of $a^{0}$, where $a^{0}$ is the equilibrium thickness of an individual layer at zero stress. According to Clark and Meyer, ${ }^{9}$ the application of a tensil force $F_{3}>0$ would induce an instability against undulations of the smectic layers. For argument's sake we assume here that this does not occur and discuss relaxation of the stress by dislocations. Whether the undulation instability occurs before dislocation effects can relax the stress depends on the times for generating the undulations in comparison with the times for dislocation nucleation and motion. In many cases the undulation effect will dominate. On the other hand, for compressional forces $F_{3}$ the Clark-Meyer instability does not occur while the dislocation effects are unchanged except for obvious reversals of signs and the sense of the dislocations.

The tensil force induces a strain $\delta D / D>0$. So long as $\delta D<\frac{1}{2} a$ dislocations are energetically unfavorable and $F_{3}=\pi R_{0}^{2} b(\delta D / D)$. If $\delta D>\frac{1}{2} a$, the elastic energy of the system would be lowered by the introduction of an extra layer. For example, imagine that a dislocation loop like that shown in Fig. 13(a) was to nucleate at the boundary. According to Eq. (8.24) with

$$
F_{3}=\left(\pi R_{0}^{2}\right)\left[a b(2 D)^{-1}-\gamma(a R)^{-1}\right]
$$

or using Eq. (8.19)

$$
\begin{aligned}
& F_{3} \approx\left(\pi R_{0}^{2}\right) a b\left[(2 D)^{-1}-R^{-1}\right], \\
& f_{\rho}=a^{2} b D^{-1}\left[\left(R / R_{0}\right)^{2}-1\right] .
\end{aligned}
$$

If we assume a mobility per unit length of dislocation $\nu$,

$$
\dot{R}=\nu a^{2} b D^{-1}\left[\left(R / R_{0}\right)^{2}-1\right] \text {. }
$$

Assuming an initial value of $1-R / R_{0} \approx D / R_{0} \ll 1$,

$$
R / R_{0} \approx\left[1-\left(D / 2 R_{0}\right) \exp (t / \tau)\right]\left[1+\left(D / 2 R_{0}\right) \exp (t / \tau)\right]^{-1},
$$

where $\tau^{-1}=2 \nu a^{2} b\left(R_{0} D\right)^{-1}$. If one could produce homogeneous samples free of dislocations, bulk measurements to determine $b$ would have to be done at small strain $\delta D / D<a / 2 D$ or at high frequency $\omega \gg$ either $\tau^{-1}$ or the reciprocal of the time to nucleate dislocations, whichever is larger. Although we have not been able to produce a theoretical estimate of $\nu$, relaxation of the applied strain by dislocation motion requires that a definite number of molecules move into the sample from the sides. There must be an upper limit on $\stackrel{R}{R}$ of the order of the speed of sound, $R \tau^{-1}<10^{5} \mathrm{~cm} \mathrm{sec}$.

Alternatively, one could imagine a dislocation loop of the type shown in Fig. 13(b) being nucleated in the interior of the sample. In place of Eq. $(8.23)$

$$
F_{3}=-\pi R_{0}^{2} \sigma_{33}(\rho>R)-\pi R^{2} a b D^{-1}
$$

and in place of $(8.24)$

$$
f_{\rho}=a\left(\pi R_{0}^{2}\right)^{-1} F_{3}+a^{2} b D^{-1}\left[\left(R / R_{0}\right)^{2}-\frac{1}{2}\right]-\gamma R^{-1} \text {. }
$$

In this case growth of the dislocation loop is opposed by the line tension and in order for there to be unstable growth

$$
F_{3} \geqslant \pi R_{0}^{2}\left\{\gamma(R a)^{-1}+a b D^{-1}\left[\frac{1}{2}-\left(R / R_{0}\right)^{2}\right]\right\},
$$

where $R$ is the initial value for the loop. Using (8.19) and taking $R \ll R_{0}$

$$
F_{3} \geqslant \pi R_{0}^{2} a b\left[R^{-1}+(2 D)^{-1}\right] \text {. }
$$

This is now a typical nucleation and growth process, if $F_{3} \approx \pi R_{0}^{2} a b / D$ only those loops that spontaneously appear with radii of the order of $D$ will be able to grow. Others that are spontaneously generated will decay. Assume that in the absence of an external stress, a loop of radius $R$ has energy $2 \pi \gamma R \approx 2 \pi b a^{2} R$. If this is set equal to $k T$, the mean radius $R \sim k T\left(2 \pi b a^{2}\right)^{-1}<10^{-10} \mathrm{~cm}$. It appears that generation of dislocation loops by thermal fluctuations alone will not be a significant factor unless $b$ is much smaller than the values observed by Liao et $a l .{ }^{28}$ This might occur for some materials near the smectic-to-nematic phase transition.

As a practical matter is is probably very difficult to attain the ideal smectic sample described above. In all likelihood typical samples will not be perfectly uniform
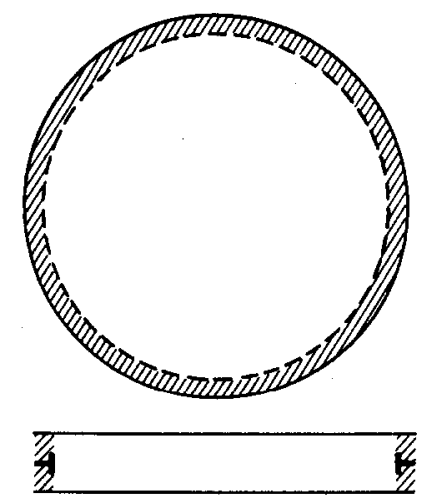

(a)

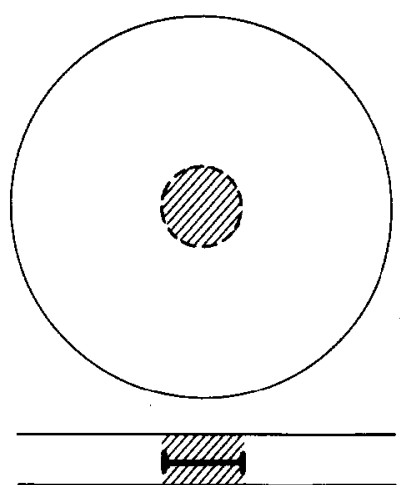

(b)
FIG. 13. Geometry of two possible dislocation loops that could spontaneously appear and relax an applied tensile stress. (a) Dislocation loop surrounding the sample with extra layer external to the loop. (b) Dislocation loop interior to the sample with extra layer within the loop. 
and dislocation loops will be pinned inside by a variety of defects. Consider, for example, a sample with a single dislocation loop of radius $R$ trapped inside. Equation (8.22) applies except that $\sigma_{33}(\rho>R)-\sigma_{33}(\rho<R)$ are no longer necessarily given by Eq. (8.18)。External forces $F_{3}$ will cause these loops to grow or shrink and the preceding analysis is probably applicable as a crude approximation. For the example of Fig. 13(a) we should replace the sample radii $R_{0}$ by a length characteristic of the sample inhomogeneity and for the analysis applicable to Fig. 13(b) that length probably corresponds to the initial value of the dislocation loop. The most important difference, however, will be that the inhomogeneous sample will be highly strained and values of $\delta D$ $<\frac{1}{2} a$ will cause the local strain in different regions to exceed $a / 2 D$. For inhomogeneous samples the simple relation $F_{3}=b \delta D / D$ probably fails for all values of $\delta D$. On the other hand, at high frequencies it can still be applicable.

\section{SCREW DISLOCATIONS}

Although Eqs. (2.3b) and (2.8) can be used to solve for the strain fields surrounding a screw dislocation, the formalism described earlier cannot be used to calculate the forces on or between screw dislocations. Equation (2.10), which described the force on an edge dislocation, is derived from the total elastic energy of the smectic using the principle of virtual work。For a given virtual displacement of a dislocation, one assumes that the change in elastic energy is dominated by the strain energy far from the dislocation core. For this to be true the core of the dislocation must be sufficiently "stiff" that it is not changed by small changes in the stresses far from the core. The energy of interaction between the core and the far field must also be negligible compared to the elastic energy far from the core. Unfortunately, the elastic energy of a screw dislocation is dominated by either the core region or the boundary forces that maintain the dislocation.

To illustrate this consider the screw dislocation depicted in Fig. 14. According to the sign convention discussed in Sec. II, the dislocation can be described by a vector

$$
1=-\hat{3} \delta\left(x_{1}\right) \delta\left(x_{2}\right)
$$

and the nonvanishing parts of Eqs。(2.3b) and $(2.8)$ are

$$
\begin{aligned}
& \partial_{2} \delta m_{1}-\partial_{1} \delta m_{2}=\delta\left(x_{1}\right) \delta\left(x_{2}\right), \\
& \nabla^{2}(\nabla \cdot m)=0
\end{aligned}
$$

where the operators $\nabla^{2}$ and $\nabla$ are two dimensional. The only solution to Eqs. (9.2) consistent with the cylindrical symmetry of the screw dislocation is

$$
\begin{aligned}
& \delta m_{1}=(2 \pi)^{-1} x_{2}\left(x_{1}^{2}+x_{2}^{2}\right)^{-1}, \\
& \delta m_{2}=-(2 \pi)^{-1} x_{1}\left(x_{1}^{2}+x_{2}^{2}\right)^{-1} .
\end{aligned}
$$

It is straightforward to demonstrate that for this solution $\nabla \cdot \delta \mathrm{m}=0$ and the stress fields $\phi=0$. The energy density given by Eqs. (2.6) is zero everywhere away from the dislocation core and the force exerted by one screw dislocation on another $[\mathrm{Eq} .(2.10)]$ is predicted to be zero. Consider now Eq. (2.6) for the elastic energy density. As we remarked earlier these expressions are not the most general that could be written. For example, consider addition of a term like

$$
\frac{1}{2} K^{*} a^{2}\left[\left(\partial_{1} m_{1}-\partial_{2} m_{2}\right)^{2}+\left(\partial_{1} m_{2}+\partial_{2} m_{1}\right)^{2}\right]
$$

or with $m_{i}=a^{-1} \partial_{i} \mu$

$$
\frac{1}{2} K^{*}\left\{\left[\left(\partial_{11}-\partial_{22}\right) \mu\right]^{2}+4\left(\partial_{12} \mu\right)^{2}\right\} \text {. }
$$

Since $\partial_{1} m_{2}=\partial_{2} m_{1}$ away from the dislocation core, Eqs. (9.4) differ from Eqs. (2.6) only by total derivatives and physical consequences of this difference can be expressed as a surface energy. The addition of Eqs. (9.4) to Eqs. (2.6) would be equivalent to assuming some specific form for the energy of the surface surrounding the core and that assumption may or may not be correct. Nevertheless, whether one adds Eqs. (9.4) to the energy density or not, the equations for the strain field are basically unchanged.

It is worth noting that substitution of the strains, Eq. (9.3), into Eqs. (9.4) gives positive definite energies. Further, if Eq. (9.4a) is included in the expression for the energy density, a nonzero term

$$
K^{*} a\left(\partial_{11}+\partial_{22}\right) \delta m_{1}
$$

must be added to the expression for $\phi_{1}[\mathrm{Eq}$. (2.7)]. Although it is tempting to simply add this term to the energy density and proceed to calculate forces, etc., we do not believe it can be justified without a better understanding of the core.

The spatial Fourier transforms of Eqs. (9.5) are

$$
\begin{aligned}
& m_{1}(q)=i q_{2} q^{-2}, \\
& m_{2}(q)=-i q_{1} q^{-2} .
\end{aligned}
$$

Inhomogeneities in the optical dielectric constants $\delta \epsilon_{13}$ and $\delta \epsilon_{23}$ which are proportional to $\delta m_{1}$ and $\delta m_{2}$ are expected to be large and light-scattering cross sections can be estimated from Eqs. (9.6). Since the screw dislocation is uniform in the $x_{3}$ direction, the scattering wave vector $\mathrm{K}$ must obey the selection rule

$$
K_{3}=\left(\mathbf{k}^{\mathrm{scat}}-\mathbf{k}^{\mathrm{inc}}\right)_{3}=0 \text {. }
$$

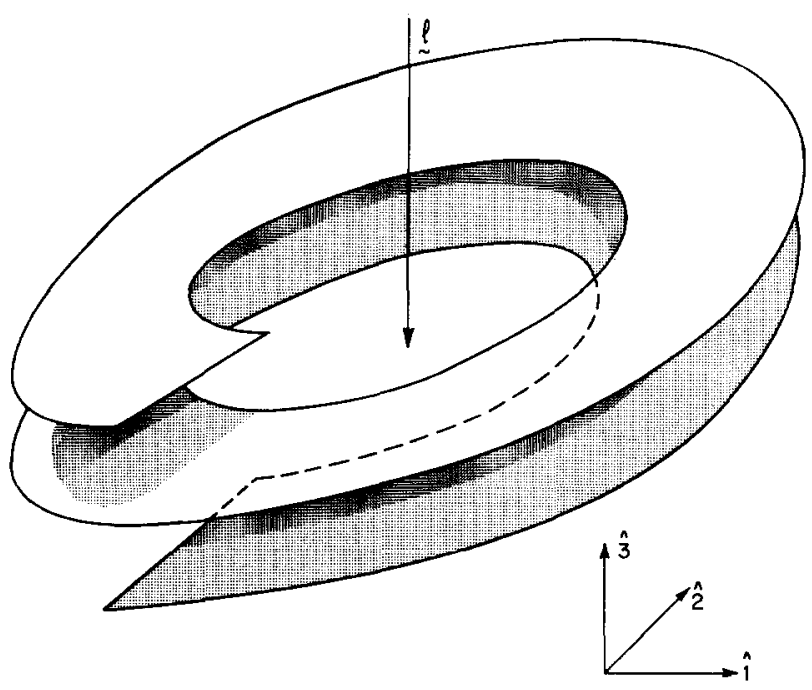

FIG, 14, Illustration of the distortion of smectic layers around a screw dislocation. According to the sign convention discussed in the text, the vector I associated with this dislocation is down. 
This selection rule was approximately satisfied for scattering from edge dislocations. In contrast for the screw dislocation $\delta \epsilon_{13} \sim q_{2}$ and $\delta \epsilon_{23} \sim q_{1}$, while for the edge dislocation $\delta \epsilon_{13} \sim q_{1}$ and $\delta \epsilon_{23} \sim q_{2}$. Optical scattering effects that have been observed do not reflect the properties expected for screw dislocations. ${ }^{10,11}$

Finally, note that for a regular array of screw dislocations at $x_{1}=0, x_{2}=n d$, where $n=0, \pm 1, \pm 2, \ldots$, the strain pattern can be calculated from Eqs. (9.5),

$$
M_{2}=\sum_{n=-\infty}^{\infty}(2 \pi)^{-1}\left(-x_{1}\right)\left[x_{1}^{2}+\left(x_{2}-n d\right)^{2}\right]
$$

and for $x_{1} \gg d$

$$
M_{2} \approx(2 \pi d)^{-1}\left(-x_{1}\right) \int_{-\infty}^{\infty}\left(x_{1}^{2}+s^{2}\right) d s=-(2 d)^{-1} x_{1}\left|x_{1}\right|^{-1} \cdot(9.8)
$$

Since $M_{2}=-a^{-1} \partial_{2} \mu$, the smectic layers on one side of the array $\left(x_{1}>0\right)$ make an angle $\delta \theta_{2}=a / d$ with respect to those on the other side. An array of screw dislocations describe a twist wall between two domains. It would be interesting to try and observe light scattering from such a domain wall.

\section{$X$. FINAL REMARKS}

For the most part this article has dealt with idealized dislocations and boundary effects. In real samples edge dislocations will be curved and even become screwlike as one moves along the dislocation. Dislocation loops may be intertwined, and will rarely be flat and pure edgelike. One question to pose is whether or not it will be practical to say anything quantitative about the observable macroscopic properties of real samples with large densities of randomly distributed dislocations. Although we have not been able to answer the question, the analogy, discussed in Sec. II, between the magnetic problem and the smectic problem suggests one possibility for further research.If $\phi$ and $\mathrm{m}$ are analogous to $B$ and $H$ respectively, it would be tempting to describe the elastic response relating $\phi$ to $\mathrm{m}$ by methods similar to those used to treat the magnetic response of $B$ to $H$. We have already alluded to this type of analogy by speaking of the "diaelasticity" of smectics.

In the magnetic problem one begins with a microscopic $B$ field and a set of microscopic currents. The macroscopic $B$ and $H$ are obtained by averaging the microscopic equations over suitable volume elements, thereby eliminating the details of the microscopic currents. A similar approach might be profitable in the dislocation problem. If macroscopic stresses and strains can be obtained by suitable averaging of the microscopic quantities, it may be possible to relate these quantities by a response function which depends on only statistical averages of the dislocations. We discussed the simplest result of this type in relation to samples in which dislocations are easily created and free to move.

Alternatively the mechanical properties of smectics can be considered in terms of the de Gennes analogy. From the discussion in Sec. VIII regarding circular dislocation loops, one might hope to derive an expression relating $\nabla \times 1$ to the average stress in the vicinity of the loop. ${ }^{37}$ The combination of Eqs. $(2.3 b)$ and this equation gives a pair of equations analogous to the superconductor problem:

$$
\begin{aligned}
& \nabla \times \mathrm{v}_{s}=-(e / m c) \mathrm{B}, \\
& \nabla \times \mathrm{B}=\left(4 \pi n_{s} e / c\right) v_{s} .
\end{aligned}
$$

With $\mathrm{v}_{s}$ analogous to $\delta \mathrm{m}$ and $e / m c$ analogous to $\mathrm{B}$, the macroscopic mechanical properties of a smectic with dislocations may be similar to the macroscopic electrical properties of a superconductor with vortices.

In the absence of more experimental information on the actual mechanical properties of smectics, it is impossible to know whether either of the two analogies discussed here has practical application. We hope that this paper and a future one ${ }^{37}$ will help to define the experiments that should be undertaken.

The alternative analogies mentioned above aside the discussion in Sec. VIII have direct application to problems of isolated focal conic regions in the smectic samples. A circular focal conic defect in an otherwise uniform ideal smectic sample has the same symmetry as the dislocation loop discussed above and stability does not seem to be a practical difficulty. The far-field stress-strain fields arising from the focal conic can be expressed in terms of the solutions to the dislocation loop problem if the latter are regarded as Green's functions. One has only to assume boundary conditions connecting the core of the focal conic to the region outside the core in which the linear elastic theory is applicable. In this way the mutual forces between isolated focal conic defects can be estimated.

\section{ACKNOWLEDGMENTS}

The authors are grateful to Professor M. Ashby, Professor $R$. Meyer, and Professor D. Turnbull for helpful conversations relevant to this work as well as criticisms of the manuscript.

\section{APPENDIX: INTERACTION ENERGY OF A PAIR OF ISOLATED EDGE DISLOCATIONS}

On substitution of Eq. (4.1) into the expression for the energy density (2.6b), the interaction terms are only those terms containing products $\delta m_{i}^{\alpha} \delta m_{i}^{\beta}, i=1,3$. Integration over all $x_{1}, x_{3}$ should obtain $\mathbf{E q}$ 。(4.5). For simplicity take $h_{3}^{\alpha}=-h_{3}^{\beta}=\frac{1}{2} h$, and $h_{1}^{\alpha}=h_{1}^{\beta}=0$. Then using Eq. (4. 2b) it is straightforward to obtain the following for the "interaction term" in the energy density:

$$
\begin{aligned}
\epsilon^{1 \mathrm{nt}} & =2 a^{2} b \delta m_{3}^{\alpha} \delta m_{3}^{\beta} \text { if } x_{3}^{2}>(h / 2)^{2}, \\
& =0 \text { if } x_{3}^{2}<(h / 2)^{2} .
\end{aligned}
$$

For $x_{3}^{2}>(h / 2)^{2}$,

$$
\begin{aligned}
\epsilon^{\text {int }}= & a^{2} b s_{\alpha} s_{\beta}(32 \pi \lambda)^{-1}\left[x_{3}^{2}-(h / 2)^{2}\right]^{-3 / 2} x_{1}^{2} \\
& \times \exp \left(-x_{1}^{2}\left|x_{3}\right|\left\{2 \lambda\left[x_{3}^{2}-(h / 2)^{2}\right]\right\}^{-1}\right)
\end{aligned}
$$

which integrates to

$$
E^{1 \mathrm{nt}}=2 \int_{h_{3} / 2}^{\infty} d x_{3} \int_{-\infty}^{\infty} d x_{1} \epsilon^{\mathrm{tn} t}=\frac{1}{4} a^{2} b s_{\alpha} s_{\beta}(\lambda / \pi h)^{1 / 2} .
$$

From Eq. (4.4) $E^{\text {tnt }}=\psi(0, h)$. 
*Work supported in part by the Joint Services Electronics Program (U.S. Army, U.S. Navy, and U.S. Air Force) under Contract No. N00014-67-A-0298-0006 and by the National Science Foundation under Grants No. GH-33576 and GH-34401. ${ }^{1}$ For an extensive review of the field up to 1967 , see F.R.N. Nabarro, Theory of Crystal Dislocations (Clarendon, Oxford, England, 1967).

${ }^{2} \mathrm{~A}$ concise review of the early literature on liquid crystals is given by I. G. Chistyakov, Sov. Phys. -Usp. 9, 551 (1967).

${ }^{3}$ J. Friedel and M. Kleman, J. Phys. (Paris) Suppl. C4, 30, 43 (1969).

${ }^{4}$ Y. Bouligand, J. Phys. (Paris) 33, 525 (1972).

${ }^{5}$ P.C. Martin, O. Parodi, and P.S. Pershan, Phys. Rev. A 6, 2401 (1972).

${ }^{6}$ H. Schmidt and J. Jähnig, Ann. Phys. (New York) 71, 129 (1972).

${ }^{7}$ P. G. de Gennes, Solid State Commun. 10, 753 (1972).

${ }^{8} \mathrm{See}$ the comment at the conclusion of Appendix C of Ref. 5 regarding the analogy between vortices in superfluids and dislocations in smectics.

${ }^{9}$ Noel A. Clark and Robert B. Meyer, Appl. Phys. Lett. 22, 493 (1973)

${ }^{10}$ Noel A. Clark and P. S. Pershan, Phys. Rev. Lett. 30, 3 (1973).

${ }^{11}$ R. Ribotta, G. Durand, and D. Litster, Solid State Commun. 12, 27 (1973).

${ }^{12}$ Georges Durand, C. R. Acad. Sci. Paris B 275, 629 (1972).

${ }^{13}$ P. G. de Gennes, C. R. Acad. Sci. Paris B 275, 939 (1972).

${ }^{14}$ M. Peach and J.S. Koehler, Phys. Rev. 80, 436 (1950).

${ }^{15}$ This result is reviewed by Nabarro in Sec. 2.3 of Ref. 1.

${ }^{16}$ Implicit here, as in all macroscopic theories of elasticity, is the fact that $\int_{P\left(r_{1}, r_{2}\right)}|\delta \mathbf{r}| \gg a$, the thickness of a single lay$\mathrm{er}$, so that the lack of precision in how one accounts for fractional parts of a layer is unimportant.

${ }^{17} \mathrm{Again}$ we only treat macroscopic phenomena and $\mathrm{m}(r)$ is really determined by suitable averages over a number of layers. The theory being developed here does not describe the region "near" to the dislocation.

${ }^{18} \mathrm{See}$, for example, Ref. 5 , Sec. III. Here we will use the letter $\mu$ to specify the variable called $x$ there.

${ }^{19}$ See Eq. (2.19a) of Ref. 5. A canonical change of independent variables from $\rho, \mathrm{v}, \mu$ (or $x$ ), and $S$ to $p, \mathrm{v}, \mu, T$ will give Eq. $(2.5 \mathrm{a})$ on the assumption that $\mathrm{v}=\mathbf{0}$.

${ }^{20}$ P. G. deGennes, J. Phys. Suppl. C4, 30, 65 (1969).

${ }^{21}$ Although additional terms could possibly be added to $\mathrm{Eq}$. (2.6a), they can all be converted to surface terms and need not be included here. See, for example, Sec. IX, Eqs. (9.4).
${ }^{22}$ L. Landau and E. Lifshitz, Electrodynamics of Continuous Media (Addison-Wesley, Reading, Mass., 1960).

${ }^{23}$ See Sec. 2.3 of Ref. 1.

${ }^{24}$ M. Peach and J.S. Koehler, Phys. Rev. 80, 436 (1950).

${ }^{25} \mathrm{~J}$. Weertman, Philos. Mag. 11, 1217 (1965).

${ }^{26}$ The pressure is defined in Ref. 5 to be the partial derivative of the chemical potential with respect to mass density at constant temperature and layer spacing. The "stress tensor" has not been explicitly defined since it is somewhat arbitrary.

Any choice of stress tensor $\sigma_{i j}$ that gives the same force density, $\dot{g}_{i}=-\partial_{j} \sigma_{i j}$ is physically acceptable.

${ }^{27} \mathrm{This}$ potential is only applicable for an extended medium in which boundary effects are neglected.

${ }^{28}$ York Liao, Noel A. Clark, and P.S. Pershan, Phys. Rev. Lett. 30, 639 (1973).

${ }^{29}$ See Sec. 11.1. 1 of Ref. 1. This is only a rough approximation since it does not take into account the line tension or core energy per unit length of the dislocation. More precisely, one must compare $\psi$ to $k T / \xi$, where $\xi$ is a characteristic length along the dislocation. If two points on a single dislocation line are separated by a distance less than $\xi$, the motions of the two points are assumed to be highly correlated. In practice $\xi$ depends on both the core energy density and geometric factors related to boundary conditions. Thus $k T / a$ $>k T / \xi$ and the region in which thermal effects dominate is smaller than that shown.

${ }^{30}$ See Sec. 2.3.2 of Ref. 1 .

${ }^{31}$ This argument was first suggested to the author by Robert B. Meyer (private communication).

${ }^{32}$ Alternatively if Eq. (2.3b) is regarded as analogous to the London equation $\left[\nabla \times J_{s}=-\left(n_{s} e^{2} / m c\right)\right.$ B], one has de Gennes's (Ref. 7) analogy to superconductors. The motion of dislocations in smectics is then analogous to the motion of vortices in superfluids. See, for example, Y.B. Kim and M.J. Stephen, in Superconductivity, edited by R.D. Parks (Marcel Dekker, New York, 1969), p. 1107.

${ }^{33}$ Noel Clark and Robert B. Meyer (private communication). See, however, Robert B. Meyer and P.S. Pershan, Solid State Commun. 13, 989 (1973).

${ }^{34}$ M. Delaye, R. Ribotta, and G. Durand, Phys. Lett. A 44, 139 (1973).

${ }^{35}$ Bateman Project, Tables of Integral Transforms, Vol. II (McGraw-Hill, New York, 1954), see Table 8.11, No. 23.

${ }^{36}$ Bateman Project, Tables of Integral Transforms, Vol. II (McGraw-Hill, New York, 1954), Table 8. 3, No. 18.

${ }^{37}$ This idea results from discussions with J. Prost and will be discussed at greater length in a subsequent publication. 